\title{
GLOBAL WELL-POSEDNESS OF THE $3 D$ PRIMITIVE EQUATIONS WITH PARTIAL VERTICAL TURBULENCE MIXING HEAT DIFFUSION
}

\author{
CHONGSHENG CAO AND EDRISS S. TITI
}

\begin{abstract}
The three-dimensional incompressible viscous Boussinesq equations, under the assumption of hydrostatic balance, govern the large scale dynamics of atmospheric and oceanic motion, and are commonly called the primitive equations. To overcome the turbulence mixing a partial vertical diffusion is usually added to the temperature advection (or density stratification) equation. In this paper we prove the global regularity of strong solutions to this model in a three-dimensional infinite horizontal channel, subject to periodic boundary conditions in the horizontal directions, and with no-penetration and stress-free boundary conditions on the solid, top and bottom, boundaries. Specifically, we show that short time strong solutions to the above problem exist globally in time, and that they depend continuously on the initial data.
\end{abstract}

MSC Subject Classifications: 35Q35, 65M70, 86-08,86A10.

Keywords: Primitive equations, Boussinesq equations, Navier-Stokes equations, turbulence mixing model, global regularity.

\section{INTRODUCTION}

The partial differential equation model that describes convective flow in ocean dynamics is known to be the Boussinesq equations, which are the Navier-Stokes equations (NSE) of incompressible flows with rotation coupled to the heat (or density stratification) and salinity transport equations. The questions of the global well-posedness of the $3 D$ Navier-Stokes equations are considered to be among the most challenging mathematical problems. In the context of the atmosphere and the ocean circulation dynamics geophysicists take advantage of the shallowness of the oceans and the atmosphere to simplify the Boussinesq equations by modeling the vertical motion with the hydrostatic balance. This leads to the well-known primitive equations for ocean and atmosphere dynamics (see, e.g., [24], [25], [29], [31], [32], [37], [38] and references therein). A vertical heat diffusivity is usually added as a leading order approximation to the effect of micro-scale turbulence mixing (cf., e.g., [16], [17], [24], [31]). As a result one arrives to the following dimensionless $3 D$ variant of the primitive equations (Boussinesq equations):

$$
\begin{aligned}
& \frac{\partial v}{\partial t}+\left(v \cdot \nabla_{H}\right) v+w \frac{\partial v}{\partial z}+f_{0} \vec{k} \times v+\nabla_{H} p+L_{1} v=0 \\
& \partial_{z} p+T=0, \\
& \nabla_{H} \cdot v+\partial_{z} w=0, \\
& \frac{\partial T}{\partial t}+v \cdot \nabla_{H} T+w \frac{\partial T}{\partial z}+L_{2} T=Q,
\end{aligned}
$$

where the horizontal velocity vector field $v=\left(v_{1}, v_{2}\right)$, the velocity vector field $\left(v_{1}, v_{2}, w\right)$, the temperature $T$ and the pressure $p$ are the unknowns. $f_{0}$ is the Coriolis parameter, $Q$ is a given heat source. For simplicity, we drop the coupling with the salinity equation, which is an advection diffusion equation, but the results reported here will be equally valid with the addition of the coupling with the salinity. Moreover, we also assume for simplicity that $Q$ is time independent. The viscosity and the heat vertical diffusion operators $L_{1}$ and $L_{2}$, respectively, are

Date: October 25, 2010. 
given by

$$
\begin{aligned}
L_{1} & =-\frac{1}{R_{1}} \Delta_{H}-\frac{1}{R_{2}} \frac{\partial^{2}}{\partial z^{2}}, \\
L_{2} & =-\frac{1}{R_{3}} \frac{\partial^{2}}{\partial z^{2}},
\end{aligned}
$$

where $R_{1}, R_{2}$ are positive constants representing the horizontal and vertical dimensionless Reynolds numbers, respectively, and $R_{3}$ is positive constant which stands for the vertical dimensionless eddy heat diffusivity turbulence mixing coefficient (cf., e.g., [16], [17]). We set $\nabla_{H}=\left(\partial_{x}, \partial_{y}\right)$ to be the horizontal gradient operator and $\Delta_{H}=\partial_{x}^{2}+\partial_{y}^{2}$ to be the horizontal Laplacian. We denote by

$$
\begin{aligned}
& \Gamma_{u}=\left\{(x, y, 0) \in \mathbb{R}^{3}\right\}, \\
& \Gamma_{b}=\left\{(x, y,-h) \in \mathbb{R}^{3}\right\},
\end{aligned}
$$

the upper and lower solid boundaries, respectively. We equip system (1)-(4), on the physical top and bottom boundaries, with the following no-normal flow and stress free boundary conditions for the flow velocity vector field $(v, w)$, namely,

$$
\begin{aligned}
& \text { on } \Gamma_{u}: \frac{\partial v}{\partial z}=0, w=0, \\
& \text { on } \Gamma_{b}: \frac{\partial v}{\partial z}=0, w=0,
\end{aligned}
$$

and for simplicity, we set the Dirichlet boundary condition for $T$ :

$$
\left.T\right|_{z=0}=0,\left.\quad T\right|_{z=-h}=1 .
$$

Horizontally, we set $(v, w)$ and $T$ to satisfy periodic boundary conditions:

$$
\begin{aligned}
& v(x+1, y, z)=v(x, y+1, z)=v(x, y, z) \\
& w(x+1, y, z)=w(x, y+1, z)=w(x, y, z) ; \\
& T(x+1, y, z)=T(x, y+1, z)=T(x, y, z) .
\end{aligned}
$$

We will denote by

$$
M=(0,1)^{2} \quad \text { and } \quad \Omega=M \times(-h, 0) .
$$

In addition, we supply the system with the initial condition:

$$
\begin{aligned}
& v(x, y, z, 0)=v_{0}(x, y, z), \\
& T(x, y, z, 0)=T_{0}(x, y, z) .
\end{aligned}
$$

System (1)-(16) is a modified form of the rotational Rayleigh-Bénard convection problem taking into consideration the geophysical situation of the shallowness of oceans and atmosphere. The original three-dimensional RayleighBénard convection model (which is identical, in the absence of heat diffusion, to the Boussinesq model of stratified fluid) has been a subject to study for many years, numerically, experimentally and analytically (see, e.g., [3], [8], [12], [18], [28], [29], [30], [37], and references therein). However, the question of global regularity is still open and is as challenging as the $3 D$ NSE. Recently, the authors of $[9]$ and [20] have shown the global well-posedness to the $2 D$ Boussinesq equations without diffusivity in the heat transport equation (see also, [11], for recent improvement). In [7] it is observed that thanks to hydrostatic balance (2) the unknown pressure is essentially a function of the two-dimensional horizontal variables. We take advantage of this observation in [7] to establish the $L^{6}$ estimates for the velocity vector field which allows us to prove the global well-posedness of the $3 D$ primitive equations under the geophysical boundary conditions. In [22] the authors take advantage of this observation as well, and proved the global well-posedness of the $3 D$ primitive equations with the Dirichlet boundary conditions by dealing directly with the "pressure" which is a function of two variables. In this paper we study system (1)-(16), exploring again the hydrostatic balance which leads to an unknown "pressure" that is a function of only two variables, and use 
the techniques and ideas introduced in [7], [9] and [20], to show in section 3 that strong solutions exist globally in time provided they exist for a short interval of time. Furthermore, we show in section 4 the uniqueness and continuous dependence on initial data of these strong solutions. The short time existence of strong solutions to this model will be reported in a forthcoming paper.

This paper is organized as follows. In section 2, we reformulate system (1)-(16) and introduce our notations and recall some well-known inequalities. Section 3 is the main section in which we establish the required estimates for proving the global existence in time for any initial data. In section 4 we prove the uniqueness of the solutions and their continuous dependence on initial data.

\section{Functional Setting and Formulation}

2.1. Equivalent Formulation. We denote by

$$
\bar{\phi}(x, y)=\frac{1}{h} \int_{-h}^{0} \phi(x, y, z) d z, \quad \forall(x, y) \in M ;
$$

and denote the fluctuation by

$$
\widetilde{\phi}=\phi-\bar{\phi}
$$

Notice that

$$
\overline{\widetilde{\phi}}=0 .
$$

Similar to [7], by integrating (2) and (3) vertically, we get

$$
w(x, y, z, t)=-\int_{-h}^{z} \nabla_{H} \cdot v(x, y, \xi, t) d \xi,
$$

and

$$
p(x, y, z, t)=-\int_{-h}^{z} T(x, y, \xi, t) d \xi+p_{s}(x, y, t),
$$

where $p_{s}$ is the pressure on the bottom $z=-h$. Essentially, $p_{s}(x, y, t)$ is the unknown pressure, and we observe, as before, that it is a function of two spatial variables $(x, y)$. As we mentioned in the introduction we explore this property as in [7] (see also [22]) to prove our global regularity result.

Replacing $T$ by $T+\frac{z}{h}$, we have the following equivalent formulation for system (1)-(16):

$$
\begin{aligned}
& \frac{\partial v}{\partial t}+L_{1} v+\left(v \cdot \nabla_{H}\right) v-\left(\int_{-h}^{z} \nabla_{H} \cdot v(x, y, \xi, t) d \xi\right) \frac{\partial v}{\partial z} \\
& \quad+f_{0} \vec{k} \times v+\nabla_{H} p_{s}(x, y, t)-\nabla_{H} \int_{-h}^{z} T(x, y, \xi, t) d \xi=0 \\
& \nabla_{H} \cdot \bar{v}=0 \\
& \frac{\partial T}{\partial t}+L_{2} T+v \cdot \nabla_{H} T-\left(\int_{-h}^{z} \nabla_{H} \cdot v(x, y, \xi, t) d \xi\right)\left(\frac{\partial T}{\partial z}+\frac{1}{h}\right)=Q, \\
& \left.\frac{\partial v}{\partial z}\right|_{z=0}=\left.\frac{\partial v}{\partial z}\right|_{z=-h}=0, \quad v(x+1, y, z)=v(x, y+1, z)=v(x, y, z), \\
& \left.T\right|_{z=0}=\left.T\right|_{z=-h}=0, \quad T(x+1, y, z)=T(x, y+1, z)=T(x, y, z), \\
& v(x, y, z, 0)=v_{0}(x, y, z), \\
& T(x, y, z, 0)=T_{0}(x, y, z)-\frac{z}{h} .
\end{aligned}
$$


In addition, $\bar{v}$ and $\widetilde{v}$ satisfy the following coupled system of equations:

$$
\begin{aligned}
& \frac{\partial \bar{v}}{\partial t}-\frac{1}{R_{1}} \Delta_{H} \bar{v}+\left(\bar{v} \cdot \nabla_{H}\right) \bar{v}+\overline{\left[\left(\widetilde{v} \cdot \nabla_{H}\right) \widetilde{v}+\left(\nabla_{H} \cdot \widetilde{v}\right) \widetilde{v}\right]} \\
& +f_{0} \vec{k} \times \bar{v}+\nabla_{H}\left[p_{s}(x, y, t)-\frac{1}{h} \int_{-h}^{0} \int_{-h}^{z} T(x, y, \xi, t) d \xi d z\right]=0, \\
& \nabla_{H} \cdot \bar{v}=0, \\
& \frac{\partial \widetilde{v}}{\partial t}+L_{1} \widetilde{v}+\left(\widetilde{v} \cdot \nabla_{H}\right) \widetilde{v}-\left(\int_{-h}^{z} \nabla_{H} \cdot \widetilde{v}(x, y, \xi, t) d \xi\right) \frac{\partial \widetilde{v}}{\partial z}+\left(\widetilde{v} \cdot \nabla_{H}\right) \bar{v}+\left(\bar{v} \cdot \nabla_{H}\right) \widetilde{v} \\
& -\overline{\left[\left(\widetilde{v} \cdot \nabla_{H}\right) \widetilde{v}+\left(\nabla_{H} \cdot \widetilde{v}\right) \widetilde{v}\right.}+f_{0} \vec{k} \times \widetilde{v}-\nabla_{H}\left(\int_{-h}^{z} T(x, y, \xi, t) d \xi-\frac{1}{h} \int_{-h}^{0} \int_{-h}^{z} T(x, y, \xi, t) d \xi d z\right)=0 .
\end{aligned}
$$

2.2. Functional spaces and inequalities. Let us denote by $L^{q}(\Omega), L^{q}(M)$ and $W^{m, q}(\Omega), W^{m, q}(M)$, and $H^{m}(\Omega)=: W^{m, 2}(\Omega), H^{m}(M)=: W^{m, 2}(M)$, the usual $L^{q}$-Lebesgue and Sobolev spaces, respectively $([1])$. We denote by

$$
\|\phi\|_{q}= \begin{cases}\left(\int_{\Omega}|\phi(x, y, z)|^{q} d x d y d z\right)^{\frac{1}{q}}, & \text { for every } \phi \in L^{q}(\Omega) \\ \left(\int_{M}|\phi(x, y)|^{q} d x d y\right)^{\frac{1}{q}}, & \text { for every } \phi \in L^{q}(M) .\end{cases}
$$

For convenience, we recall the following Sobolev and Ladyzhenskaya type inequalities in $(\mathbb{R} / \mathbb{Z})^{2}$ and in $\Omega$ (see, e.g., [1], [10], [15], [23])

$$
\begin{aligned}
& \|\phi\|_{L^{4}(M)} \leq C_{0}\|\phi\|_{L^{2}}^{1 / 2}\|\phi\|_{H^{1}(M)}^{1 / 2}, \quad \forall \phi \in H^{1}(M), \\
& \|\phi\|_{L^{8}(M)} \leq C_{0}\|\phi\|_{L^{6}(M)}^{3 / 4}\|\phi\|_{H^{1}(M)}^{1 / 4}, \quad \forall \phi \in H^{1}(M), \\
& \left\|\nabla_{H} \phi\right\|_{L^{4}(M)} \leq C_{0}\|\phi\|_{\infty}^{1 / 2}\|\phi\|_{H^{2}(M)}^{1 / 2}, \quad \forall \phi \in H^{2}(M), \\
& \left\|\nabla_{H} \phi\right\|_{L^{4}(M)} \leq C_{0}\|\phi\|_{L^{2}(M)}^{1 / 2}\left\|\nabla_{H} \phi\right\|_{\infty}^{1 / 2}+\|\phi\|_{L^{2}(M)}, \quad \forall \phi \text { such that } \nabla_{H} \phi \in L^{\infty}(M),
\end{aligned}
$$

and

$$
\begin{aligned}
& \|\psi\|_{L^{3}(\Omega)} \leq C_{0}\|\psi\|_{L^{2}(\Omega)}^{1 / 2}\|\psi\|_{H^{1}(\Omega)}^{1 / 2}, \\
& \|\psi\|_{L^{6}(\Omega)} \leq C_{0}\|\psi\|_{H^{1}(\Omega)},
\end{aligned}
$$

for every $\psi \in H^{1}(\Omega)$. Here $C_{0}$ is a positive scale invariant constant. Also, we recall the following version of Helmholtz-Weyl decomposition Theorem (cf. for example, [26], [15], [39])

$$
\left\|\nabla_{H} \phi\right\|_{W^{m, q}(M)} \leq C\left(\left\|\nabla_{H} \cdot \phi\right\|_{W^{m, q}(M)}+\left\|\nabla_{H}^{\perp} \cdot \phi\right\|_{W^{m, q}(M)}\right),
$$

for every $\vec{\phi} \in\left(W^{m, q}(M)\right)^{2}$. Moreover, we recall the following Brezis-Gallouet or, Brezis-Wainger inequality (see, e.g., [26], [4], [5], [13])

$$
\|\phi\|_{L^{\infty}(M)} \leq C\|\phi\|_{H^{1}(M)}\left(1+\log ^{+}\|\phi\|_{H^{2}(M)}\right)^{1 / 2},
$$

for every $\phi \in H^{2}(M)$, where $\log ^{+} r=\log r$, when $r \geq 1$, and $\log ^{+} r=0$, when $r \leq 1$. Also, we recall the following inequality (see, e.g., [2] and [21])

$$
\left\|\nabla_{H} \phi\right\|_{L^{\infty}(M)} \leq C\left(\left\|\nabla_{H} \cdot \phi\right\|_{L^{\infty}(M)}+\left\|\nabla_{H} \times \phi\right\|_{L^{\infty}(M)}\right)\left(1+\log ^{+}\left\|\nabla_{H} \phi\right\|_{H^{2}(M)}\right),
$$

for every $\nabla_{H} \phi \in H^{2}(M)$. Moreover, by (33) we get

$$
\begin{aligned}
& \|\phi\|_{L^{4 q}(M)}^{4 q}=\left\||\phi|^{q}\right\|_{L^{4}(M)}^{4} \leq C\left\||\phi|^{q}\right\|_{L^{2}(M)}^{2}\left\||\phi|^{q}\right\|_{H^{1}(M)}^{2} \\
& \leq C_{q}\|\phi\|_{2 q}^{2 q}\left(\int_{M}|\phi|^{2 q-2}\left|\nabla_{H} \phi\right|^{2} d x d y\right)+\|\phi\|_{2 q}^{4 q},
\end{aligned}
$$


for every $\phi$ satisfying $\int_{M}|\phi|^{2 q-2}\left|\nabla_{H} \phi\right|^{2} d x d y<\infty$ and $q \geq 1$. Also, we recall the integral version of Minkowsky inequality for the $L^{p}$ spaces, $p \geq 1$. Let $\Omega_{1} \subset \mathbb{R}^{m_{1}}$ and $\Omega_{2} \subset \mathbb{R}^{m_{2}}$ be two measurable sets, where $m_{1}$ and $m_{2}$ are two positive integers. Suppose that $f(\xi, \eta)$ is a measurable function over $\Omega_{1} \times \Omega_{2}$. Then,

$$
\left[\int_{\Omega_{1}}\left(\int_{\Omega_{2}}|f(\xi, \eta)| d \eta\right)^{p} d \xi\right]^{1 / p} \leq \int_{\Omega_{2}}\left(\int_{\Omega_{1}}|f(\xi, \eta)|^{p} d \xi\right)^{1 / p} d \eta
$$

Finally, we recall the following inequality from Proposition 2.2 in [6]

$$
\begin{aligned}
& \left|\int_{M}\left(\int_{-h}^{0} \psi_{1}(x, y, z) d z\right)\left(\int_{-h}^{0} \psi_{2}(x, y, z) \psi_{3}(x, y, z) d z\right) d x d y\right| \\
& \leq C\left\|\psi_{1}\right\|_{2}^{1 / 2}\left\|\nabla_{H} \psi_{1}\right\|_{2}^{1 / 2}\left\|\psi_{2}\right\|_{2}^{1 / 2}\left\|\nabla_{H} \psi_{2}\right\|_{2}^{1 / 2}\left\|\psi_{3}\right\|_{2}+\left\|\psi_{1}\right\|_{2}\left\|\psi_{2}\right\|_{2}\left\|\psi_{3}\right\|_{2},
\end{aligned}
$$

for every $\psi_{1}, \psi_{2} \in H^{1}(\Omega)$ and $\psi_{3} \in L^{2}(\Omega)$, and

$$
\begin{aligned}
& \left|\int_{M}\left(\int_{-h}^{0} \psi_{1}(x, y, z) d z\right)\left(\int_{-h}^{0}\left|\nabla_{H} \psi_{2}(x, y, z)\right| \psi_{3}(x, y, z) d z\right) d x d y\right| \\
& \leq C\left\|\psi_{1}\right\|_{2}^{1 / 2}\left\|\nabla_{H} \psi_{1}\right\|_{2}^{1 / 2}\left\|\psi_{2}\right\|_{\infty}^{1 / 2}\left\|\nabla_{H} \nabla_{H} \psi_{2}\right\|_{2}^{1 / 2}\left\|\psi_{3}\right\|_{2}+\left\|\psi_{1}\right\|_{2}\left\|\psi_{2}\right\|_{2}\left\|\psi_{3}\right\|_{2},
\end{aligned}
$$

for every $\psi_{1} \in H^{1}(\Omega), \nabla_{H} \psi_{2} \in H^{1}(\Omega)$ and $\psi_{3} \in L^{2}(\Omega)$.

\section{Global existence of Strong solutions}

In the previous section we have reformulated system (1)-(16) to be equivalent to system (22)-(28). In this section we will show that strong solutions to system (22)-(28) exist globally in time provided they exist in short time intervals.

Theorem 1. Let $Q \in H^{2}(\Omega), v_{0} \in H^{4}(\Omega), T_{0} \in H^{2}(\Omega)$ and $\mathcal{T}>0$. Suppose that there exists a strong solution $(v(t), T(t))$ of system $(22)-(28)$ on $[0, \mathcal{T}]$ corresponding to the initial data $\left(v_{0}, T_{0}\right)$ such that

$$
\begin{aligned}
& \Delta_{H} v_{z}, \quad \nabla_{H} T \in C\left([0, \mathcal{T}], H^{1}(\Omega)\right), \\
& v_{z z}, \Delta_{H} \nabla_{H} v_{z}, \quad \nabla_{H} T_{z} \in L^{2}\left([0, \mathcal{T}], H^{1}(\Omega)\right) .
\end{aligned}
$$

Then this strong solution $(v(t), T(t))$ exists globally in time.

Remark 1. Notice that one can recover the pressure $p_{s}$ from system (29)-(30) in the same way as in $2 D$ NSE (see, e.g., [10], [34], [35]).

Proof. Let $\left[0, \mathcal{T}_{*}\right)$ be the maximal interval of existence of a strong solution $(v(t), T(t))$. In order to establish the global existence, we need to show That $\mathcal{T}_{*}=\infty$. If $\mathcal{T}_{*}<\infty$ we will show $\left\|\Delta_{H} v_{z}(t)\right\|_{H^{1}(\Omega)}, \quad\left\|\nabla_{H} T(t)\right\|_{H^{1}(\Omega)}$, $\int_{0}^{t}\left\|\Delta_{H} \nabla_{H} v_{z}(s)\right\|_{H^{1}(\Omega)}^{2} d s, \int_{0}^{t}\left\|\nabla_{H} T_{z}(s)\right\|_{H^{1}(\Omega)}^{2} d s$, and $\int_{0}^{t}\left\|v_{z z}(s)\right\|_{H^{1}(\Omega)}^{2} d s$ are all bounded uniformly in time, for $t \in\left[0, \mathcal{T}_{*}\right)$. As a result the interval $\left[0, \mathcal{T}_{*}\right)$ can not be a maximal interval of existence, and consequently the strong solution $(v(t), T(t))$ exists globally in time.

Therefore, we focus our discussion below on the interval $\left[0, \mathcal{T}_{*}\right)$.

3.1. $\|v\|_{2}^{2}+\|T\|_{2}^{2}$ estimates. By taking the inner product of equation (24) with $T$, in $L^{2}(\Omega)$, we get

$$
\begin{aligned}
& \frac{1}{2} \frac{d\|T\|_{2}^{2}}{d t}+\frac{1}{R_{3}}\left\|T_{z}\right\|_{2}^{2} \\
& =\int_{\Omega} Q T d x d y d z-\int_{\Omega}\left[v \cdot \nabla_{H} T-\left(\int_{-h}^{z} \nabla_{H} \cdot v(x, y, \xi, t) d \xi\right)\left(\frac{\partial T}{\partial z}+\frac{1}{h}\right)\right] T d x d y d z .
\end{aligned}
$$

Integrating by parts and using the boundary condition (26), we get

$$
-\int_{\Omega}\left(v \cdot \nabla_{H} T-\left(\int_{-h}^{z} \nabla_{H} \cdot v(x, y, \xi, t) d \xi\right) \frac{\partial T}{\partial z}\right) T d x d y d z=0 .
$$


As a result of the above we conclude that

$$
\begin{aligned}
& \frac{1}{2} \frac{d\|T\|_{2}^{2}}{d t}+\frac{1}{R_{3}}\left\|T_{z}\right\|_{2}^{2} \\
& =\int_{\Omega}\left[Q-\frac{1}{h}\left(\int_{-h}^{z} \nabla_{H} \cdot v(x, y, \xi, t) d \xi\right)\right] T d x d y d z \leq\|Q\|_{2}\|T\|_{2}+\left\|\nabla_{H} v\right\|_{2}\|T\|_{2} .
\end{aligned}
$$

Moreover, by taking the inner product of equation (22) with $v$, in $L^{2}(\Omega)$, we reach

$$
\begin{aligned}
\frac{1}{2} & \frac{d\|v\|_{2}^{2}}{d t}+\frac{1}{R_{1}}\left\|\nabla_{H} v\right\|_{2}^{2}+\frac{1}{R_{2}}\left\|v_{z}\right\|_{2}^{2} \\
= & -\int_{\Omega}\left[\left(v \cdot \nabla_{H}\right) v-\left(\int_{-h}^{z} \nabla_{H} \cdot v(x, y, \xi, t) d \xi\right) \frac{\partial v}{\partial z}\right] \cdot v d x d y d z \\
& -\int_{\Omega}\left(f_{0} \vec{k} \times v\right) \cdot v d x d y d z-\int_{\Omega}\left(\nabla_{H} p_{s}-\nabla_{H}\left(\int_{-h}^{z} T(x, y, \xi, t) d \xi\right)\right) \cdot v d x d y d z .
\end{aligned}
$$

First, we notice that

$$
\left(f_{0} \vec{k} \times v\right) \cdot v=0 .
$$

Next, by integration by parts and using the boundary conditions (25), in particular, the horizontal periodic boundary conditions, we get

$$
\int_{\Omega}\left[\left(v \cdot \nabla_{H}\right) v-\left(\int_{-h}^{z} \nabla_{H} \cdot v(x, y, \xi, t) d \xi\right) \frac{\partial v}{\partial z}\right] \cdot v d x d y d z=0 .
$$

Thanks to (30) and, again, the horizontal periodic boundary conditions, we also have

$$
\int_{\Omega} \nabla_{H} p_{s}(x, y, t) \cdot v(x, y, z, t) d x d y d z=h \int_{M} \nabla_{H} p_{s} \cdot \bar{v} d x d y=-h \int_{\Omega} p_{s}\left(\nabla_{H} \cdot \bar{v}\right) d x d y=0 .
$$

By integration by parts, the periodic boundary conditions (25), and Cauchy-Schwarz inequality, we obtain

$$
\left|\int_{\Omega} \nabla_{H}\left(\int_{-h}^{z} T(x, y, \xi, t) d \xi\right) \cdot v d x d y d z\right| \leq h\|T\|_{2}\left\|\nabla_{H} v\right\|_{2} .
$$

Thus, by (48)-(53) we have

$$
\begin{aligned}
& \frac{1}{2} \frac{d\left(\|v\|_{2}^{2}+\|T\|_{2}^{2}\right)}{d t}+\frac{1}{R_{1}}\left\|\nabla_{H} v\right\|_{2}^{2}+\frac{1}{R_{2}}\left\|v_{z}\right\|_{2}^{2}+\frac{1}{R_{3}}\left\|T_{z}\right\|_{2}^{2} \\
& \leq\|Q\|_{2}\|T\|_{2}+(1+h)\|T\|_{2}\left\|\nabla_{H} v\right\|_{2} .
\end{aligned}
$$

By Cauchy-Schwarz inequality, we obtain

$$
\begin{aligned}
& \frac{d\left(\|v\|_{2}^{2}+\|T\|_{2}^{2}\right)}{d t}+\frac{1}{R_{1}}\left\|\nabla_{H} v\right\|_{2}^{2}+\frac{1}{R_{2}}\left\|v_{z}\right\|_{2}^{2}+\frac{1}{R_{3}}\left\|T_{z}\right\|_{2}^{2} \\
& \leq\|Q\|_{2}^{2}+\left(1+R_{1}\right)(1+h)^{2}\|T\|_{2}^{2} .
\end{aligned}
$$

Thanks to Gronwall's inequality we get

$$
\|v(t)\|_{2}^{2}+\|T(t)\|_{2}^{2} \leq C\left(\left\|v_{0}\right\|_{2}^{2}+\left\|T_{0}\right\|_{2}^{2}\right) e^{\left(1+R_{1}\right)(1+h)^{2} t}+C\|Q\|_{2}^{2}
$$

and

$$
\int_{0}^{t}\left[\frac{1}{R_{1}}\left\|\nabla_{H} v(s)\right\|_{2}^{2}+\frac{1}{R_{2}}\left\|v_{z}(s)\right\|_{2}^{2}+\frac{1}{R_{3}}\left\|T_{z}(s)\right\|_{2}^{2}\right] d s \leq C\left[\left(\left\|v_{0}\right\|_{2}^{2}+\left\|T_{0}\right\|_{2}^{2}\right) e^{\left(1+R_{1}\right)(1+h)^{2} t}+\|Q\|_{2}^{2} t\right] .
$$


Therefore, for every $t \in\left[0, \mathcal{T}_{*}\right)$, we have

$$
\|v(t)\|_{2}^{2}+\|T(t)\|_{2}^{2}+\int_{0}^{t}\left[\left\|\nabla_{H} v(s)\right\|_{2}^{2}+\left\|v_{z}(s)\right\|_{2}^{2}+\left\|T_{z}(s)\right\|_{2}^{2}\right] d s \leq K_{1},
$$

where

$$
K_{1}=C\left[\left(\left\|v_{0}\right\|_{2}^{2}+\left\|T_{0}\right\|_{2}^{2}\right) e^{\left(1+R_{1}\right)(1+h)^{2} t}+\|Q\|_{2}^{2} t\right] .
$$

3.2. $\|T\|_{\infty}$ estimates. We follow here the idea of Stampaccia for proving the Maximum Principle. The proof we present here is also similar to the one in [14] (see also [36]). Denote by $\tau(t)=T(t)-\left(1+\left\|T_{0}\right\|_{\infty}+\|Q\|_{\infty} t\right.$ ). It is clear that $\tau$ satisfies:

$$
\frac{\partial \tau}{\partial t}+v \cdot \nabla_{H} \tau+w \frac{\partial \tau}{\partial z}+L_{2} \tau=Q-\|Q\|_{\infty}
$$

Let $\tau^{+}=\max \{0, \tau\}$ which belongs to $H^{1}(\Omega)$ and satisfies

$$
\tau^{+}(z=0)=\tau^{+}(z=-h)=0 .
$$

Taking the inner product of the equation (61) with $\tau^{+}$in $L^{2}(\Omega)$ and applying the boundary conditions (62), we get

$$
\frac{1}{2} \frac{d\left\|\tau^{+}\right\|_{2}^{2}}{d t}+\frac{1}{R_{3}}\left\|\partial_{z} \tau^{+}\right\|_{2}^{2}=\int_{\Omega}\left(Q-\|Q\|_{\infty}\right) \tau^{+} d x d y d z-\int_{\Omega}\left[v \cdot \nabla_{H} \tau+w \partial_{z} \tau\right] \tau^{+} d x d y d z .
$$

By integration by parts and using the boundary conditions (26) and (62), we get

$$
\int_{\Omega}\left[v \cdot \nabla_{H} \tau+w \partial_{z} \tau\right] \tau^{+} d x d y d z=0
$$

Thus,

$$
\frac{1}{2} \frac{d\left\|\tau^{+}\right\|_{2}^{2}}{d t}+\frac{1}{R_{3}}\left\|\partial_{z} \tau^{+}\right\|_{2}^{2} d x d y d z=\int_{\Omega}\left(Q-\|Q\|_{\infty}\right) \tau^{+} d x d y d z \leq 0 .
$$

Therefore, we obtain

$$
\left\|\tau^{+}(t)\right\|_{2}^{2} \leq\left\|\tau^{+}(t=0)\right\|_{2}^{2}=0 .
$$

Thus, $\tau^{+}(t) \equiv 0$. As a result, we have

$$
T(t) \leq 1+\left\|T_{0}\right\|_{\infty}+\|Q\|_{\infty} t .
$$

By applying similar arguments, we also have

$$
T(t) \geq-\left(1+\left\|T_{0}\right\|_{\infty}+\|Q\|_{\infty} t\right) .
$$

Therefore, $T$ satisfies the following $L^{\infty}$-estimate:

$$
\|T(t)\|_{\infty} \leq K_{2}=1+\left\|T_{0}\right\|_{\infty}+\|Q\|_{\infty} t .
$$


3.3. $\|\widetilde{v}\|_{6}$ estimates. Taking the inner product of the equation (31) with $|\widetilde{v}|^{4} \widetilde{v}$ in $L^{2}(\Omega)$ and using the boundary conditions (25), we get

$$
\begin{aligned}
& \frac{1}{6} \frac{d\|\widetilde{v}\|_{6}^{6}}{d t}+\frac{1}{R_{1}} \int_{\Omega}\left(\left|\nabla_{H} \widetilde{v}\right|^{2}|\widetilde{v}|^{4}+\left.\left.\left|\nabla_{H}\right| \widetilde{v}\right|^{2}\right|^{2}|\widetilde{v}|^{2}\right) d x d y d z+\frac{1}{R_{2}} \int_{\Omega}\left(\left|\widetilde{v}_{z}\right|^{2}|\widetilde{v}|^{4}+\left.\left.\left|\partial_{z}\right| \widetilde{v}\right|^{2}\right|^{2}|\widetilde{v}|^{2}\right) d x d y d z \\
& =-\int_{\Omega}\left\{\left(\widetilde{v} \cdot \nabla_{H}\right) \widetilde{v}-\left(\int_{-h}^{z} \nabla_{H} \cdot \widetilde{v}(x, y, \xi, t) d \xi\right) \frac{\partial \widetilde{v}}{\partial z}+\left(\widetilde{v} \cdot \nabla_{H}\right) \bar{v}+\left(\bar{v} \cdot \nabla_{H}\right) \widetilde{v}-\overline{\left[\left(\widetilde{v} \cdot \nabla_{H}\right) \widetilde{v}+\left(\nabla_{H} \cdot \widetilde{v}\right) \widetilde{v}\right]}\right. \\
& \left.+f_{0} \vec{k} \times \widetilde{v}-\nabla_{H}\left(\int_{-h}^{z} T(x, y, \xi, t) d \xi-\frac{1}{h} \int_{-h}^{0} \int_{-h}^{z} T(x, y, \xi, t) d \xi d z\right)\right\} \cdot|\widetilde{v}|^{4} \widetilde{v} d x d y d z .
\end{aligned}
$$

Observe, again, that

$$
\left(f_{0} \vec{k} \times \widetilde{v}\right) \cdot|\widetilde{v}|^{4} \widetilde{v}=0 .
$$

Moreover, by integration by parts and the boundary conditions (25), we also get

$$
-\int_{\Omega}\left[\left(\widetilde{v} \cdot \nabla_{H}\right) \widetilde{v}-\left(\int_{-h}^{z} \nabla_{H} \cdot \widetilde{v}(x, y, \xi, t) d \xi\right) \frac{\partial \widetilde{v}}{\partial z}\right] \cdot|\widetilde{v}|^{4} \widetilde{v} d x d y d z=0 .
$$

Furthermore, by virtue of (30) and by the boundary conditions (25), in particular the horizontal periodic boundary conditions, we have

$$
\int_{\Omega}\left(\bar{v}(x, y, t) \cdot \nabla_{H}\right) \widetilde{v}(x, y, z, t) \cdot|\widetilde{v}(x, y, z, t)|^{4} \widetilde{v}(x, y, z, t) d x d y d z=0 .
$$

Thus, (70)-(72) imply

$$
\begin{aligned}
& \frac{1}{6} \frac{d\|\widetilde{v}\|_{6}^{6}}{d t}+\frac{1}{R_{1}} \int_{\Omega}\left(\left|\nabla_{H} \widetilde{v}\right|^{2}|\widetilde{v}|^{4}+\left.\left.\left|\nabla_{H}\right| \widetilde{v}\right|^{2}\right|^{2}|\widetilde{v}|^{2}\right) d x d y d z+\frac{1}{R_{2}} \int_{\Omega}\left(\left|\widetilde{v}_{z}\right|^{2}|\widetilde{v}|^{4}+\left.\left.\left|\partial_{z}\right| \widetilde{v}\right|^{2}\right|^{2}|\widetilde{v}|^{2}\right) d x d y d z \\
& =-\int_{\Omega}\left\{\left(\widetilde{v} \cdot \nabla_{H}\right) \bar{v}-\overline{\left(\widetilde{v} \cdot \nabla_{H}\right) \widetilde{v}+\left(\nabla_{H} \cdot \widetilde{v}\right) \widetilde{v}}\right. \\
& \left.\quad-\nabla_{H}\left(\int_{-h}^{z} T(x, y, \xi, t) d \xi-\frac{1}{h} \int_{-h}^{0} \int_{-h}^{z} T(x, y, \xi, t) d \xi d z\right\}\right) \cdot|\widetilde{v}|^{4} \widetilde{v} d x d y d z .
\end{aligned}
$$

Notice that by integration by parts and using the boundary conditions (25), in particular the horizontal periodic boundary conditions, we have

$$
\begin{aligned}
- & \int_{\Omega}\left[\left(\widetilde{v} \cdot \nabla_{H}\right) \bar{v}-\overline{\left[\left(\widetilde{v} \cdot \nabla_{H}\right) \widetilde{v}+\left(\nabla_{H} \cdot \widetilde{v}\right) \widetilde{v}\right]}\right. \\
& \left.-\nabla_{H}\left(\int_{-h}^{z} T(x, y, \xi, t) d \xi-\frac{1}{h} \int_{-h}^{0} \int_{-h}^{z} T(x, y, \xi, t) d \xi d z\right)\right] \cdot|\widetilde{v}|^{4} \widetilde{v} d x d y d z \\
= & \int_{\Omega}\left[\left(\nabla_{H} \cdot \widetilde{v}\right) \bar{v} \cdot|\widetilde{v}|^{4} \widetilde{v}+\left(\widetilde{v} \cdot \nabla_{H}\right)\left(|\widetilde{v}|^{4} \widetilde{v}\right) \cdot \bar{v}-\sum_{k=1}^{2} \sum_{j=1}^{3} \overline{\widetilde{v}^{k} \widetilde{v} \widetilde{v}^{j}} \partial_{x_{k}}\left(|\widetilde{v}|^{4} \widetilde{v}^{j}\right)\right. \\
& \left.-\left(\int_{-h}^{z} T(x, y, \xi, t) d \xi-\frac{1}{h} \int_{-h}^{0} \int_{-h}^{z} T(x, y, \xi, t) d \xi d z\right) \nabla_{H} \cdot\left(|\widetilde{v}|^{4} \widetilde{v}\right)\right] d x d y d z .
\end{aligned}
$$


As a result, we obtain

$$
\begin{aligned}
& \frac{1}{6} \frac{d\|\widetilde{v}\|_{6}^{6}}{d t}+\frac{1}{R_{1}} \int_{\Omega}\left(\left|\nabla_{H} \widetilde{v}\right|^{2}|\widetilde{v}|^{4}+\left.\left.\left|\nabla_{H}\right| \widetilde{v}\right|^{2}\right|^{2}|\widetilde{v}|^{2}\right) d x d y d z+\frac{1}{R_{2}} \int_{\Omega}\left(\left|\widetilde{v}_{z}\right|^{2}|\widetilde{v}|^{4}+\left.\left.\left|\partial_{z}\right| \widetilde{v}\right|^{2}\right|^{2}|\widetilde{v}|^{2}\right) d x d y d z \\
& \leq C \int_{M}\left[|\bar{v}| \int_{-h}^{0}\left|\nabla_{H} \widetilde{v}\right||\widetilde{v}|^{5} d z\right] d x d y+C \int_{M}\left[\left(\int_{-h}^{0}|\widetilde{v}|^{2} d z\right)\left(\int_{-h}^{0}\left|\nabla_{H} \widetilde{v}\right||\widetilde{v}|^{4} d z\right)\right] d x d y \\
& +C \int_{M}\left[\left.\left|\overline{T \mid} \int_{-h}^{0}\right| \nabla_{H} \widetilde{v}|| \widetilde{v}\right|^{4} d z\right] d x d y .
\end{aligned}
$$

Therefore, by the Cauchy-Schwarz inequality and Hölder inequality we reach

$$
\begin{aligned}
& \frac{1}{6} \frac{d\|\widetilde{v}\|_{6}^{6}}{d t}+\frac{1}{R_{1}} \int_{\Omega}\left(\left|\nabla_{H} \widetilde{v}\right|^{2}|\widetilde{v}|^{4}+\left.\left.\left|\nabla_{H}\right| \widetilde{v}\right|^{2}\right|^{2}|\widetilde{v}|^{2}\right) d x d y d z+\frac{1}{R_{2}} \int_{\Omega}\left(\left|\widetilde{v}_{z}\right|^{2}|\widetilde{v}|^{4}+\left.\left.\left|\partial_{z}\right| \widetilde{v}\right|^{2}\right|^{2}|\widetilde{v}|^{2}\right) d x d y d z \\
& \leq C \int_{M}\left[|\bar{v}|\left(\int_{-h}^{0}\left|\nabla_{H} \widetilde{v}\right|^{2}|\widetilde{v}|^{4} d z\right)^{1 / 2}\left(\int_{-h}^{0}|\widetilde{v}|^{6} d z\right)^{1 / 2}\right] d x d y \\
& +C \int_{M}\left[\left(\int_{-h}^{0}|\widetilde{v}|^{2} d z\right)\left(\int_{-h}^{0}\left|\nabla_{H} \widetilde{v}\right|^{2}|\widetilde{v}|^{4} d z\right)^{1 / 2}\left(\int_{-h}^{0}|\widetilde{v}|^{4} d z\right)^{1 / 2}\right] d x d y \\
& +C \int_{M}\left[\overline{|T|}\left(\int_{-h}^{0}\left|\nabla_{H} \widetilde{v}\right|^{2}|\widetilde{v}|^{4} d z\right)^{1 / 2}\left(\int_{-h}^{0}|\widetilde{v}|^{4} d z\right)^{1 / 2}\right] d x d y
\end{aligned}
$$

Moreover,

$$
\begin{aligned}
& \frac{1}{6} \frac{d\|\widetilde{v}\|_{6}^{6}}{d t}+\frac{1}{R_{1}} \int_{\Omega}\left(\left|\nabla_{H} \widetilde{v}\right|^{2}|\widetilde{v}|^{4}+\left.\left.\left|\nabla_{H}\right| \widetilde{v}\right|^{2}\right|^{2}|\widetilde{v}|^{2}\right) d x d y d z+\frac{1}{R_{2}} \int_{\Omega}\left(\left|\widetilde{v}_{z}\right|^{2}|\widetilde{v}|^{4}+\left.\left.\left|\partial_{z}\right| \widetilde{v}\right|^{2}\right|^{2}|\widetilde{v}|^{2}\right) d x d y d z \\
& \leq C\|\bar{v}\|_{L^{4}(M)}\left(\int_{\Omega}\left|\nabla_{H} \widetilde{v}\right|^{2}|\widetilde{v}|^{4} d x d y d z\right)^{1 / 2}\left(\int_{M}\left(\int_{-h}^{0}|\widetilde{v}|^{6} d z\right)^{1 / 4} d x d y\right)^{1 / 4}\left(\int_{\Omega}\left|\nabla_{H} \widetilde{v}\right|^{2}|\widetilde{v}|^{4} d x d y d z\right)^{1 / 2}\left(\int_{M}\left(\int_{-h}^{0}|\widetilde{v}|^{4} d z\right)^{2} d x d y\right)^{1 / 4} \\
& \quad+C\left(\int_{M}\left(\int_{-h}^{0}|\widetilde{v}|^{2} d z\right)^{4} d x d y\right)^{1 / 4}\left(\int_{M}\left(\int_{-h}^{0}|\widetilde{v}|^{4} d z\right)^{2} d x d y\right)^{.2} .
\end{aligned}
$$

Using the Minkowsky inequality (43) we get

$$
\left(\int_{M}\left(\int_{-h}^{0}|\widetilde{v}|^{6} d z\right)^{2} d x d y\right)^{1 / 2} \leq C \int_{-h}^{0}\left(\int_{M}|\widetilde{v}|^{12} d x d y\right)^{1 / 2} d z
$$

Thanks to (42),

$$
\int_{M}|\widetilde{v}|^{12} d x d y \leq C_{0}\left(\int_{M}|\widetilde{v}|^{6} d x d y\right)\left(\int_{M}|\widetilde{v}|^{4}\left|\nabla_{H} \widetilde{v}\right|^{2} d x d y\right)+\left(\int_{M}|\widetilde{v}|^{6} d x d y\right)^{2} .
$$

Thus, by the Cauchy-Schwarz inequality we obtain

$$
\left(\int_{M}\left(\int_{-h}^{0}|\widetilde{v}|^{6} d z\right)^{2} d x d y\right)^{1 / 2} \leq C\|\widetilde{v}\|_{L^{6}(\Omega)}^{3}\left(\int_{\Omega}|\widetilde{v}|^{4}\left|\nabla_{H} \widetilde{v}\right|^{2} d x d y d z\right)^{1 / 2}+\|\widetilde{v}\|_{L^{6}(\Omega)}^{6}
$$


Similarly, by (43) and (34), we also obtain

$$
\begin{aligned}
& \left(\int_{M}\left(\int_{-h}^{0}|\widetilde{v}|^{4} d z\right)^{2} d x d y\right)^{1 / 2} \leq C \int_{-h}^{0}\left(\int_{M}|\widetilde{v}|^{8} d x d y\right)^{1 / 2} d z \\
& \leq C \int_{-h}^{0}\|\widetilde{v}\|_{L^{6}(M)}^{3}\left(\left\|\nabla_{H} \widetilde{v}\right\|_{L^{2}(M)}+\|\widetilde{v}\|_{L^{2}(M)}\right) d z \leq C\|\widetilde{v}\|_{6}^{3}\left(\left\|\nabla_{H} \widetilde{v}\right\|_{2}+\|\widetilde{v}\|_{2}\right),
\end{aligned}
$$

and

$$
\begin{aligned}
& \left(\int_{M}\left(\int_{-h}^{0}|\widetilde{v}|^{2} d z\right)^{4} d x d y\right)^{1 / 4} \leq C \int_{-h}^{0}\left(\int_{M}|\widetilde{v}|^{8} d x d y\right)^{1 / 4} d z \\
& \leq C \int_{-h}^{0}\|\widetilde{v}\|_{L^{6}(M)}^{3 / 2}\left(\left\|\nabla_{H} \widetilde{v}\right\|_{L^{2}(M)}^{1 / 2}+\|\widetilde{v}\|_{L^{2}(M)}^{1 / 2}\right) d z \leq C\|\widetilde{v}\|_{6}^{3 / 2}\left(\left\|\nabla_{H} \widetilde{v}\right\|_{2}+\|\widetilde{v}\|_{2}\right)^{1 / 2} .
\end{aligned}
$$

Therefore, using (81)-(83) and (33), we reach to

$$
\begin{aligned}
& \frac{1}{6} \frac{d\|\widetilde{v}\|_{6}^{6}}{d t}+\frac{1}{R_{1}} \int_{\Omega}\left(\left|\nabla_{H} \widetilde{v}\right|^{2}|\widetilde{v}|^{4}+\left.\left.\left|\nabla_{H}\right| \widetilde{v}\right|^{2}\right|^{2}|\widetilde{v}|^{2}\right) d x d y d z+\frac{1}{R_{2}} \int_{\Omega}\left(|\widetilde{v}|^{2}|\widetilde{v}|^{4}+\left.\left.\left|\partial_{z}\right| \widetilde{v}\right|^{2}\right|^{2}|\widetilde{v}|^{2}\right) d x d y d z \\
& \leq C\|\bar{v}\|_{2}^{1 / 2}\left\|\nabla_{H} \bar{v}\right\|_{2}^{1 / 2}\|\widetilde{v}\|_{6}^{3 / 2}\left(\int_{\Omega}\left|\nabla_{H} \widetilde{v}\right|^{2}|\widetilde{v}|^{4} d x d y d z\right)^{3 / 4}+C\|\widetilde{v}\|_{6}^{3}\left(\left\|\nabla_{H} \widetilde{v}\right\|_{2}+\|\widetilde{v}\|_{2}\right)\left(\int_{\Omega}\left|\nabla_{H} \widetilde{v}\right|^{2}|\widetilde{v}|^{4} d x d y d z\right)^{1 / 2} \\
& +C\|\bar{v}\|_{2}^{1 / 2}\left\|\nabla_{H} \bar{v}\right\|_{2}^{1 / 2}\|\widetilde{v}\|_{6}^{6}+C\|T\|_{\infty}\|\widetilde{v}\|_{6}^{3 / 2}\left(\left\|\nabla_{H} \widetilde{v}\right\|_{2}^{1 / 2}+\|\widetilde{v}\|_{2}^{1 / 2}\right)\left(\int_{\Omega}\left|\nabla_{H} \widetilde{v}\right|^{2}|\widetilde{v}|^{4} d x d y d z\right)^{1 / 2} .
\end{aligned}
$$

By Young's inequality and Cauchy-Schwarz inequality we have

$$
\begin{aligned}
& \frac{d\|\widetilde{v}\|_{6}^{6}}{d t}+\frac{1}{R_{1}} \int_{\Omega}\left(\left|\nabla_{H} \widetilde{v}\right|^{2}|\widetilde{v}|^{4}+\left.\left.\left|\nabla_{H}\right| \widetilde{v}\right|^{2}\right|^{2}|\widetilde{v}|^{2}\right) d x d y d z+\frac{1}{R_{2}} \int_{\Omega}\left(\left|\widetilde{v}_{z}\right|^{2}|\widetilde{v}|^{4}+\left.\left.\left|\partial_{z}\right| \widetilde{v}\right|^{2}\right|^{2}|\widetilde{v}|^{2}\right) d x d y d z \\
& \leq C\|\bar{v}\|_{2}^{2}\left\|\nabla_{H} \bar{v}\right\|_{2}^{2}\|\widetilde{v}\|_{6}^{6}+C\|\widetilde{v}\|_{6}^{6}\left\|\nabla_{H} \widetilde{v}\right\|_{2}^{2}+C\|T\|_{\infty}^{4}+C\|\widetilde{v}\|_{2}^{2}\|\widetilde{v}\|_{6}^{6} .
\end{aligned}
$$

Thanks to (59), (69) and Gronwall inequality, we get

$$
\|\widetilde{v}(t)\|_{6}^{6}+\int_{0}^{t}\left(\frac{1}{R_{1}} \int_{\Omega}\left|\nabla_{H} \widetilde{v}\right|^{2}|\widetilde{v}|^{4} d x d y d z+\frac{1}{R_{2}} \int_{\Omega}\left|\widetilde{v}_{z}\right|^{2}\left|\widetilde{v}_{z}\right|^{4} d x d y d z\right) \leq K_{3},
$$

where

$$
K_{3}=e^{K_{1}^{2} t}\left[\left\|v_{0}\right\|_{H^{1}(\Omega)}^{6}+K_{2}^{4} t\right] .
$$

3.4. $\left\|\nabla_{H} \bar{v}\right\|_{2}$ estimates. By taking the inner product of equation (29) with $-\Delta_{H} \bar{v}$ in $L^{2}(M)$, and applying (30), and using the boundary conditions (25), we reach

$$
\frac{1}{2} \frac{d\left\|\nabla_{H} \bar{v}\right\|_{2}^{2}}{d t}+\frac{1}{R_{1}}\left\|\Delta_{H} \bar{v}\right\|_{2}^{2}=\int_{M}\left\{\left(\bar{v} \cdot \nabla_{H}\right) \bar{v}+\overline{\left[\left(\widetilde{v} \cdot \nabla_{H}\right) \widetilde{v}+\left(\nabla_{H} \cdot \widetilde{v}\right) \widetilde{v}\right.}+f_{0} \vec{k} \times \bar{v}\right\} \cdot \Delta_{H} \bar{v} d x d y .
$$

Following the situation for the $2 D$ Navier-Stokes equations (cf. e.g., [10], [32]) we have

$$
\left|\int_{M}\left(\bar{v} \cdot \nabla_{H}\right) \bar{v} \cdot \Delta_{H} \bar{v} d x d y\right| \leq C\|\bar{v}\|_{2}^{1 / 2}\left\|\nabla_{H} \bar{v}\right\|_{2}\left\|\Delta_{H} \bar{v}\right\|_{2}^{3 / 2} .
$$


By the Cauchy-Schwarz and the Hölder inequalities, we have

$$
\begin{aligned}
& \left|\int_{M} \overline{\left(\widetilde{v} \cdot \nabla_{H}\right) \widetilde{v}+\left(\nabla_{H} \cdot \widetilde{v}\right)} \widetilde{v} \cdot \Delta_{H} \bar{v} d x d y\right| \leq C \int_{M} \int_{-h}^{0}|\widetilde{v}|\left|\nabla_{H} \widetilde{v}\right| d z\left|\Delta_{H} \bar{v}\right| d x d y \\
& \leq C \int_{M}\left[\left(\int_{-h}^{0}|\widetilde{v}|^{2}\left|\nabla_{H} \widetilde{v}\right| d z\right)^{1 / 2}\left(\int_{-h}^{0}\left|\nabla_{H} \widetilde{v}\right| d z\right)^{1 / 2}\left|\Delta_{H} \bar{v}\right|\right] d x d y \\
& \leq C\left[\int_{M}\left(\int_{-h}^{0}|\widetilde{v}|^{2}\left|\nabla_{H} \widetilde{v}\right| d z\right)^{2} d x d y\right]^{1 / 4}\left[\int_{M}\left(\int_{-h}^{0}\left|\nabla_{H} \widetilde{v}\right| d z\right)^{2} d x d y\right]^{1 / 4}\left[\int_{M}\left|\Delta_{H} \bar{v}\right|^{2} d x d y\right]^{1 / 2} \\
& \leq C\left\|\nabla_{H} \widetilde{v}\right\|_{2}^{1 / 2}\left(\int_{\Omega}|\widetilde{v}|^{4}\left|\nabla_{H} \widetilde{v}\right|^{2} d x d y d z\right)^{1 / 4}\left\|\Delta_{H} \bar{v}\right\|_{2},
\end{aligned}
$$

and

$$
\left|\int_{M} f_{0} \times \bar{v} \cdot \Delta_{H} \bar{v} d x d y\right| \leq C\|\bar{v}\|_{2}\left\|\Delta_{H} \bar{v}\right\|_{2}
$$

Thus, by Young's inequality and the Cauchy-Schwarz inequality, we have

$$
\frac{d\left\|\nabla_{H} \bar{v}\right\|_{2}^{2}}{d t}+\frac{1}{R_{1}}\left\|\Delta_{H} \bar{v}\right\|_{2}^{2} \leq C\|\bar{v}\|_{2}^{2}\left\|\nabla_{H} \bar{v}\right\|_{2}^{4}+C\left\|\nabla_{H} \widetilde{v}\right\|_{2}^{2}+C \int_{\Omega}|\widetilde{v}|^{4}\left|\nabla_{H} \widetilde{v}\right|^{2} d x d y d z+C\|\bar{v}\|_{2}^{2}
$$

By (59), (84) and thanks to Gronwall inequality we obtain

$$
\left\|\nabla_{H} \bar{v}\right\|_{2}^{2}+\frac{1}{R_{1}} \int_{0}^{t}\left|\Delta_{H} \bar{v}\right|_{2}^{2} d s \leq K_{4}
$$

where

$$
K_{4}=e^{K_{2}^{2} t}\left[\left\|v_{0}\right\|_{H^{1}(\Omega)}^{2}+K_{2}+K_{3}\right] .
$$

3.5. $\left\|v_{z}\right\|_{6}$ estimates. The a priori estimates (59)-(91) are essentially similar to those obtained in [7]. From now on, we will get new a priori estimates of various norms.

Denote by $u=v_{z}$. It is clear that $u$ satisfies

$$
\begin{aligned}
& \frac{\partial u}{\partial t}+L_{1} u+\left(v \cdot \nabla_{H}\right) u-\left(\int_{-h}^{z} \nabla_{H} \cdot v(x, y, \xi, t) d \xi\right) \frac{\partial u}{\partial z} \\
& \quad+\left(u \cdot \nabla_{H}\right) v-\left(\nabla_{H} \cdot v\right) u+f_{0} \vec{k} \times u-\nabla_{H} T=0 . \\
& \left.u\right|_{z=0}=\left.u\right|_{z=-h}=0 .
\end{aligned}
$$

Taking the inner product of the equation (93) with $u|u|^{4}$ in $L^{2}$, we get

$$
\begin{aligned}
& \frac{1}{6} \frac{d\|u\|_{6}^{6}}{d t}+\frac{5}{R_{1}}\left\||u|^{2}\left|\nabla_{H} u\right|\right\|_{2}^{2}+\frac{5}{R_{2}}\left\||u|^{2}\left|\partial_{z} u\right|\right\|_{2}^{2} \\
& =-\int_{\Omega}\left(\left(v \cdot \nabla_{H}\right) u-\left(\int_{-h}^{z} \nabla_{H} \cdot v(x, y, \xi, t) d \xi\right) \frac{\partial u}{\partial z}\right) \cdot u|u|^{4} d x d y d z \\
& \quad-\int_{\Omega}\left(\left(u \cdot \nabla_{H}\right) v-\left(\nabla_{H} \cdot v\right) u+f_{0} \vec{k} \times u-\nabla_{H} T\right) \cdot u|u|^{4} d x d y d z
\end{aligned}
$$

Notice again that

$$
f_{0} \vec{k} \times u \cdot u|u|^{4}=0
$$


Integrating by parts and using the boundary conditions, in particular (94), give

$$
-\int_{\Omega}\left(\left(v \cdot \nabla_{H}\right) u-\left(\int_{-h}^{z} \nabla_{H} \cdot v(x, y, \xi, t) d \xi\right) \frac{\partial u}{\partial z}\right) \cdot u|u|^{4} d x d y d z=0 .
$$

Thus, by (96), (97) and Hölder inequality, we have

$$
\begin{aligned}
& \frac{1}{6} \frac{d\|u\|_{6}^{6}}{d t}+\frac{5}{R_{1}}\left\||u|^{2}\left|\nabla_{H} u\right|\right\|_{2}^{2}+\frac{5}{R_{2}}\left\||u|^{2}\left|\partial_{z} u\right|\right\|_{2}^{2} \\
& =-\int_{\Omega}\left(\left(u \cdot \nabla_{H}\right) v-\left(\nabla_{H} \cdot v\right) u-\nabla_{H} T\right) \cdot u|u|^{4} d x d y d z \\
& \leq C \int_{\Omega}|v||u|^{5}\left|\nabla_{H} u\right| d x d y d z+C \int_{\Omega}|T||u|^{4}\left|\nabla_{H} u\right| d x d y d z \\
& \leq C\left(\|v\|_{6}\left\|u^{3}\right\|_{3}\left\|\nabla_{H} u^{2}\right\|_{2}+\|T\|_{6}\left\|u^{2}\right\|_{3}\left\|\nabla_{H} u^{3}\right\|_{2}\right) \\
& \leq C\left(\|v\|_{6}\|u\|_{6}^{3 / 2}\left\|\nabla_{H} u^{3}\right\|_{2}^{3 / 2}+\|T\|_{6}\|u\|_{6}^{2}\left\|\nabla_{H} u^{3}\right\|_{2}\right) .
\end{aligned}
$$

Thanks to the Cauchy-Schwarz inequality, we have

$$
\begin{aligned}
& \frac{d\|u\|_{6}^{6}}{d t}+\frac{1}{R_{1}}\left\||u|^{2}\left|\nabla_{H} u\right|\right\|_{2}^{2}+\frac{1}{R_{2}}\left\||u|^{2}\left|\partial_{z} u\right|\right\|_{2}^{2} \\
& \leq C\left(1+\|v\|_{6}^{4}\right)\|u\|_{6}^{6}+\|T\|_{6}^{6} \\
& \leq C\left(1+\left\|\nabla_{H} \bar{v}\right\|_{2}^{4}+\|\widetilde{v}\|_{6}^{4}\right)\|u\|_{6}^{6}+\|T\|_{6}^{6} .
\end{aligned}
$$

Using (59), (84), (91), and Gronwall inequality, we get

$$
\|u\|_{6}^{6}+\int_{0}^{t}\left[\frac{1}{R_{1}}\left\||u|^{2}\left|\nabla_{H} u\right|\right\|_{2}^{2}+\frac{1}{R_{2}}\left\||u|^{2}\left|\partial_{z} u\right|\right\|_{2}^{2}\right] d s \leq K_{5},
$$

where

$$
K_{5}=e^{\left(1+K_{3}^{2 / 3}+K_{4}^{2}\right) t}\left[\left\|\partial_{z} v_{0}\right\|_{H^{1}(\Omega)}^{6}+K_{2}^{6} t\right] .
$$

3.6. $\left\|v_{z z}\right\|_{2}$ estimates. Taking the inner product of the equation (93) with $-u_{z z}$ in $L^{2}(\Omega)$ and recalling that $u=v_{z}$, which satisfies the boundary condition (94), we get

$$
\begin{aligned}
\frac{1}{2} & \frac{d\left\|u_{z}\right\|_{2}^{2}}{d t}+\frac{1}{R_{1}}\left\|\nabla_{H} u_{z}\right\|_{2}^{2}+\frac{1}{R_{2}}\left\|u_{z z}\right\|_{2}^{2} \\
& =\int_{\Omega}\left(\left(v \cdot \nabla_{H}\right) u-\left(\int_{-h}^{z} \nabla_{H} \cdot v(x, y, \xi, t) d \xi\right) \frac{\partial u}{\partial z}\right) \cdot u_{z z} d x d y d z \\
& +\int_{\Omega}\left(\left(u \cdot \nabla_{H}\right) v-\left(\nabla_{H} \cdot v\right) u+f_{0} \vec{k} \times u-\nabla_{H} T\right) \cdot u_{z z} d x d y d z \\
& =-\int_{\Omega}\left[\left(u \cdot \nabla_{H}\right) u+\left(u_{z} \cdot \nabla_{H}\right) v+\left(u \cdot \nabla_{H}\right) u-\left(\nabla_{H} \cdot u\right) u-\left(\nabla_{H} \cdot v\right) u_{z}\right] \cdot u_{z} d x d y d z-\int_{\Omega} T_{z}\left(\nabla_{H} \cdot u_{z}\right) d x d y d z \\
& \leq C\|u\|_{6}\left\|\nabla_{H} u\right\|_{2}\left\|u_{z}\right\|_{3}+C\|v\|_{6}\left\|\nabla_{H} u_{z}\right\|_{2}\left\|u_{z}\right\|_{3}+\left\|T_{z}\right\|_{2}\left\|\nabla_{H} u_{z}\right\|_{2} \\
& \leq C\left[\|u\|_{6}\left\|\nabla_{H} u\right\|_{2}+\|v\|_{6}\left\|\nabla_{H} u_{z}\right\|_{2}\right]\left\|u_{z}\right\|_{2}^{1 / 2}\left(\left\|\nabla_{H} u_{z}\right\|_{2}^{1 / 2}+\left\|u_{z z}\right\|_{2}^{1 / 2}\right)+\left\|T_{z}\right\|_{2}\left\|\nabla_{H} u_{z}\right\|_{2} .
\end{aligned}
$$

By the Cauchy-Schwarz and Young's inequalities, we have

$$
\begin{aligned}
& \frac{1}{2} \frac{d\left\|u_{z}\right\|_{2}^{2}}{d t}+\frac{1}{R_{1}}\left\|\nabla_{H} u_{z}\right\|_{2}^{2}+\frac{1}{R_{2}}\left\|u_{z z}\right\|_{2}^{2} \\
& \leq C\left(\|v\|_{6}^{4}+\|u\|_{6}^{4}\right)\left\|u_{z}\right\|_{2}^{2}+C\left\|\nabla_{H} u\right\|_{2}^{2}+C\left\|T_{z}\right\|_{2}^{2} .
\end{aligned}
$$


Applying (59), (84), (101), and Gronwall inequality yield

$$
\left\|u_{z}\right\|_{2}^{2}+\int_{0}^{t}\left[\frac{1}{R_{1}}\left\|\nabla_{H} u_{z}\right\|_{2}^{2}+\frac{1}{R_{2}}\left\|u_{z z}\right\|_{2}^{2}\right] d s \leq K_{6},
$$

where

$$
K_{6}=C e^{\left(K_{3}^{2 / 3}+K_{5}^{2 / 3}\right) t}\left[\left\|v_{0}\right\|_{H^{1}(\Omega)}^{2}+K_{1}\right] .
$$

3.7. $\left\|\nabla_{H} \times v_{z}\right\|_{2}^{2}+\left\|\nabla_{H} \cdot v_{z}+R_{1} T\right\|_{2}^{2}$ estimates. Let $\beta$ be the solution of the following two-dimensional elliptic problem with periodic boundary conditions:

$$
\Delta_{H} \beta=\nabla_{H} T, \quad \int_{M} \beta d x d y=0,
$$

where $z$ is considered as a parameter. Roughly speaking, $\beta$ is like the potential vorticity. Notice that

$$
\nabla_{H} \cdot \beta=T, \quad \nabla_{H} \times \beta=0 .
$$

Recall that $u=v_{z}$. We denote by

$$
\begin{aligned}
& \zeta=u+R_{1} \beta, \\
& \eta=\left(\nabla_{H}^{\perp} \cdot \zeta\right)=\nabla_{H}^{\perp} \cdot u=\partial_{x} u_{2}-\partial_{y} u_{1}, \\
& \theta=\left(\nabla_{H} \cdot \zeta\right)=\nabla_{H} \cdot u+R_{1} T=\partial_{x} u_{1}+\partial_{y} u_{2}+R_{1} T .
\end{aligned}
$$

Applying (39) for $\zeta$ and $\beta$ with $m \geq 0,1<q<\infty$, using (106)-(109), we obtain

$$
\begin{aligned}
& \left\|\nabla_{H} u\right\|_{W^{m, q}(M)} \leq C\left(\left\|\nabla_{H} \zeta\right\|_{W^{m, q}(M)}+R_{1}\left\|\nabla_{H} \beta\right\|_{W^{m, q}(M)}\right) \\
& \leq C\left(\|\eta\|_{W^{m, q}(M)}+\|\theta\|_{W^{m, q}(M)}+\|T\|_{W^{m, q}(M)}\right),
\end{aligned}
$$

where, again, we consider $z$ as a parameter; consequently, the constant $C$ is independent of $z$. By applying the operator $\nabla_{H}^{\perp}$. to equation (93) we obtain

$$
\begin{aligned}
& \frac{\partial \eta}{\partial t}+L_{1} \eta+\nabla_{H}^{\perp} \cdot\left[\left(v \cdot \nabla_{H}\right) u-\left(\int_{-h}^{z} \nabla_{H} \cdot v(x, y, \xi, t) d \xi\right) \frac{\partial u}{\partial z}+\left(u \cdot \nabla_{H}\right) v-\left(\nabla_{H} \cdot v\right) u\right] \\
& -f_{0}\left(R_{1} T-\theta\right)=0 .
\end{aligned}
$$

Then, multiplying equation (24) by $R_{1}$ and adding to the above equation we reach

$$
\begin{aligned}
& \frac{\partial \theta}{\partial t}+L_{1} \theta+\nabla_{H} \cdot\left[\left(v \cdot \nabla_{H}\right) u-\left(\int_{-h}^{z} \nabla_{H} \cdot v(x, y, \xi, t) d \xi\right) \frac{\partial u}{\partial z}+\left(u \cdot \nabla_{H}\right) v-\left(\nabla_{H} \cdot v\right) u\right]-f_{0} \eta \\
& +R_{1}\left[v \cdot \nabla_{H} T-\left(\int_{-h}^{z} \nabla_{H} \cdot v(x, y, \xi, t) d \xi\right)\left(\frac{\partial T}{\partial z}+\frac{1}{h}\right)\right]=R_{1} Q+R_{1}\left(\frac{1}{R_{3}}-\frac{1}{R_{2}}\right) T_{z z} .
\end{aligned}
$$


Taking the inner product of equation (111) with $\eta$ in $L^{2}(\Omega)$ and the equation (112) with $\theta$ in $L^{2}(\Omega)$, integrating by parts and observing that $\left.\eta\right|_{z=0}=\left.\eta\right|_{z=-h}=\left.\theta\right|_{z=0}=\left.\theta\right|_{z=-h}=0$, we get

$$
\begin{aligned}
\frac{1}{2} & \frac{d\left(\|\eta\|_{2}^{2}+\|\theta\|_{2}^{2}\right)}{d t}+\frac{1}{R_{1}}\left(\left\|\nabla_{H} \eta\right\|_{2}^{2}+\left\|\nabla_{H} \theta\right\|_{2}^{2}\right)+\frac{1}{R_{2}}\left(\left\|\partial_{z} \eta\right\|_{2}^{2}+\left\|\partial_{z} \theta\right\|_{2}^{2}\right) \\
= & -\int_{\Omega}\left\{\nabla_{H}^{\perp} \cdot\left[\left(v \cdot \nabla_{H}\right) u-\left(\int_{-h}^{z} \nabla_{H} \cdot v(x, y, \xi, t) d \xi\right) \frac{\partial u}{\partial z}+\left(u \cdot \nabla_{H}\right) v-\left(\nabla_{H} \cdot v\right) u\right] \eta-f_{0} R_{1} T \eta\right\} d x d y d z \\
& -\int_{\Omega}\left\{\nabla_{H} \cdot\left[\left(v \cdot \nabla_{H}\right) u-\left(\int_{-h}^{z} \nabla_{H} \cdot v(x, y, \xi, t) d \xi\right) \frac{\partial u}{\partial z}+\left(u \cdot \nabla_{H}\right) v-\left(\nabla_{H} \cdot v\right) u\right] \theta\right\} d x d y d z \\
& -\int_{\Omega}\left\{R_{1}\left[v \cdot \nabla_{H} T-\left(\int_{-h}^{z} \nabla_{H} \cdot v(x, y, \xi, t) d \xi\right)\left(\frac{\partial T}{\partial z}+\frac{1}{h}\right)-Q+\left(\frac{1}{R_{3}}-\frac{1}{R_{2}}\right) T_{z z}\right] \theta\right\} d x d y d z \\
\leq & C\|Q\|_{2}\|\theta\|_{2}+C \int_{\Omega}\left(|v|\left|\nabla_{H} u\right|+|u|\left|\nabla_{H} v\right|+\left|u_{z}\right| \int_{-h}^{0}\left|\nabla_{H} \cdot v\right| d \xi\right)\left(\left|\nabla_{H} \eta\right|+\left|\nabla_{H} \theta\right|\right) d x d y d z+C\|T\|_{2}\|\eta\|_{2} \\
& +C \int_{\Omega}\left[\left|\nabla_{H} v\|T\| \theta\right|+|v||T|\left|\nabla_{H} \theta\right|+\left|T_{z}\right|\left(\int_{-h}^{0}\left|\nabla_{H} \cdot v\right| d \xi\right)|\theta|\right] d x d y d z+C\|v\|_{2}\left\|\nabla_{H} \theta\right\|_{2}+C\left\|T_{z}\right\|_{2}\left\|\theta_{z}\right\|_{2} \\
\leq & C\|Q\|_{2}\|\theta\|_{2}+C \int_{\Omega}\left(|v|\left|\nabla_{H} u\right|+|u|\left|\nabla_{H} v\right|+\left|u_{z}\right| \int_{-h}^{0}(|\theta|+|T|) d \xi\right)\left(\left|\nabla_{H} \eta\right|+\left|\nabla_{H} \theta\right|\right) d x d y d z+C\|T\|_{2}\|\eta\|_{2} \\
& +C \int_{\Omega}\left[\left|\nabla_{H} v\|T\| \theta\right|+|v \| T|\left|\nabla_{H} \theta\right|+\left|T_{z}\right|\left(\int_{-h}^{0}(|\theta|+|T|) d \xi\right)|\theta|\right] d x d y d z+C\|v\|_{2}\left\|\nabla_{H} \theta\right\|_{2}+C\left\|T_{z}\right\|_{2}\left\|\theta_{z}\right\|_{2} .
\end{aligned}
$$

Using Hölder inequality, and inequalities (44) and (110), we obtain

$$
\begin{aligned}
& \frac{1}{2} \frac{d\left(\|\eta\|_{2}^{2}+\|\theta\|_{2}^{2}\right)}{d t}+\frac{1}{R_{1}}\left(\left\|\nabla_{H} \eta\right\|_{2}^{2}+\left\|\nabla_{H} \theta\right\|_{2}^{2}\right)+\frac{1}{R_{2}}\left(\left\|\partial_{z} \eta\right\|_{2}^{2}+\left\|\partial_{z} \theta\right\|_{2}^{2}\right) \\
& \leq C\left(\|v\|_{6}\left\|\nabla_{H} u\right\|_{3}+\|u\|_{6}\left\|\nabla_{H} v\right\|_{3}+\left\|u_{z}\right\|_{2}^{\frac{1}{2}}\left\|\nabla_{H} u_{z}\right\|_{2}^{\frac{1}{2}}\|\theta\|_{2}^{\frac{1}{2}}\left\|\nabla_{H} \theta\right\|_{2}^{\frac{1}{2}}+\|T\|_{\infty}\left\|u_{z}\right\|_{2}\right)\left(\left\|\nabla_{H} \eta\right\|_{2}+\left\|\nabla_{H} \theta\right\|_{2}\right) \\
& \quad+C\|T\|_{2}\|\eta\|_{2}+C\left\|\nabla_{H} v\right\|_{2}\|T\|_{\infty}\|\theta\|_{2}+C\|v\|_{2}\|T\|_{\infty}\left\|\nabla_{H} \theta\right\|_{2}+C\left\|T_{z}\right\|_{2}\|\theta\|_{2}\left\|\nabla_{H} \theta\right\|_{2}+C\|T\|_{\infty}\left\|T_{z}\right\|_{2}\|\theta\|_{2} \\
& \quad+C\|Q\|_{2}\|\theta\|_{2}+C\|v\|_{2}\left\|\nabla_{H} \theta\right\|_{2}+C\left\|T_{z}\right\|_{2}\left\|\theta_{z}\right\|_{2} \\
& \leq C\left(\|T\|_{3}+\|\eta\|_{2}^{\frac{1}{2}}\left\|\nabla_{H} \eta\right\|_{2}^{\frac{1}{2}}+\|\theta\|_{2}^{\frac{1}{2}}\left\|\nabla_{H} \theta\right\|_{2}^{\frac{1}{2}}\right)\left(\|v\|_{6}+\|u\|_{6}\right)\left(\left\|\nabla_{H} \eta\right\|_{2}+\left\|\nabla_{H} \theta\right\|_{2}\right)+ \\
& \quad+C\left(\left\|u_{z}\right\|_{2}^{1 / 2}\left\|\nabla_{H} u_{z}\right\|_{2}^{1 / 2}\|\theta\|_{2}^{1 / 2}\left\|\nabla_{H} \theta\right\|_{2}^{1 / 2}+\|T\|_{\infty}\left\|u_{z}\right\|_{2}+\left\|T_{z}\right\|_{2}\|\theta\|_{2}+\|v\|_{2}\|T\|_{\infty}\right)\left(\left\|\nabla_{H} \eta\right\|_{2}+\left\|\nabla_{H} \theta\right\|_{2}\right) \\
& \quad+C\|T\|_{2}\|\eta\|_{2}+C\left(\left\|\nabla_{H} v\right\|_{2}\|T\|_{\infty}+\|T\|_{\infty}\left\|T_{z}\right\|_{2}\right)\|\theta\|_{2}+C\|Q\|_{2}\|\theta\|_{2}+C\|v\|_{2}\left\|\nabla_{H} \theta\right\|_{2}+C\left\|T_{z}\right\|_{2}\left\|\theta_{z}\right\|_{2} .
\end{aligned}
$$

By Young's and the Cauchy-Schwarz inequalities, we have

$$
\begin{aligned}
& \frac{d\left(\|\eta\|_{2}^{2}+\|\theta\|_{2}^{2}\right)}{d t}+\frac{1}{R_{1}}\left(\left\|\nabla_{H} \eta\right\|_{2}^{2}+\left\|\nabla_{H} \theta\right\|_{2}^{2}\right)+\frac{1}{R_{2}}\left(\left\|\partial_{z} \eta\right\|_{2}^{2}+\left\|\partial_{z} \theta\right\|_{2}^{2}\right) \\
& \leq C\left(1+\|v\|_{6}^{4}+\|u\|_{6}^{4}+\left\|T_{z}\right\|_{2}^{2}+\left\|u_{z}\right\|_{2}^{2}\left\|\nabla_{H} u_{z}\right\|_{2}^{2}\right)\left(\|\eta\|_{2}^{2}+\|\theta\|_{2}^{2}\right) \\
& \quad+C\|T\|_{2}^{2}+C\|Q\|_{2}^{2}+C\left(\|v\|_{6}^{2}+\|u\|_{6}^{2}+\left\|u_{z}\right\|_{2}^{2}+\|v\|_{2}^{2}+\left\|\nabla_{H} v\right\|_{2}^{2}+\left\|T_{z}\right\|_{2}^{2}\right)\left(1+\|T\|_{\infty}^{2}\right) .
\end{aligned}
$$

Thanks to (59), (69), (84), (101), (103), and Gronwall inequality, we have

$$
\|\eta\|_{2}^{2}+\|\theta\|_{2}^{2}+\int_{0}^{t}\left[\frac{1}{R_{1}}\left(\left\|\nabla_{H} \eta\right\|_{2}^{2}+\left\|\nabla_{H} \theta\right\|_{2}^{2}\right)+\frac{1}{R_{2}}\left(\left\|\partial_{z} \eta\right\|_{2}^{2}+\left\|\partial_{z} \theta\right\|_{2}^{2}\right)\right] d s \leq K_{7},
$$

where

$$
K_{7}=C e^{\left(K_{1}+K_{3}^{2 / 3}+K_{5}^{2 / 3}+K_{6}^{2}\right) t}\left[\left\|v_{0}\right\|_{H^{1}(\Omega)}^{2}+K_{1}+\|Q\|_{2}^{2}+K_{2}^{2}\left(K_{2}+K_{3}^{1 / 3}+K_{5}^{1 / 3}+K_{6}\right)\right] .
$$


3.8. $\left\|\Delta_{H} \bar{v}\right\|_{H^{1}(M)}^{2}+\left\|\nabla_{H}\left(\nabla_{H}^{\perp} \cdot v_{z}\right)\right\|_{H^{1}(\Omega)}^{2}+\left\|\nabla_{H}\left(\nabla_{H} \cdot v_{z}+R_{1} T\right)\right\|_{H^{1}(\Omega)}^{2}+\left\|\nabla_{H} T\right\|_{H^{1}(\Omega)}^{2}$ estimates. First, let us observe that

$$
\left|\nabla_{H} v(x, y, z)\right| \leq\left|\nabla_{H} \bar{v}(x, y)\right|+\int_{-h}^{0}\left|\nabla_{H} v_{z}(x, y, z)\right| d z .
$$

Therefore, from the above and (107), we have

$$
\begin{aligned}
& \left\|\nabla_{H} v\right\|_{\infty} \leq\left\|\nabla_{H} \bar{v}\right\|_{\infty}+\left\|\int_{-h}^{0}\left|\nabla_{H} u\right| d z\right\|_{\infty} \\
& \leq\left\|\nabla_{H} \bar{v}\right\|_{\infty}+R_{1} \int_{-h}^{0}\left\|\nabla_{H} \beta\right\|_{\infty} d z+\left\|\int_{-h}^{0}\left|\nabla_{H} \zeta\right| d z\right\|_{\infty} .
\end{aligned}
$$

By applying inequality (40) to $\nabla_{H} \bar{v}$ and $\int_{-h}^{0}\left|\nabla_{H} \zeta\right| d z$ we reach

$$
\begin{aligned}
& \left\|\nabla_{H} \bar{v}\right\|_{\infty} \leq C\left\|\nabla_{H} \bar{v}\right\|_{H^{1}(M)}\left(1+\log ^{+}\left\|\nabla_{H} \bar{v}\right\|_{H^{2}(M)}\right)^{1 / 2} \\
& \left\|\int_{-h}^{0}\left|\nabla_{H} \zeta\right| d z\right\|_{\infty} \leq C\left\|\int_{-h}^{0}\left|\nabla_{H} \zeta\right| d z\right\|_{H^{1}(M)}\left(1+\log ^{+}\left\|\int_{-h}^{0}\left|\nabla_{H} \zeta\right| d z\right\|_{H^{2}(M)}\right)^{1 / 2}
\end{aligned}
$$

Applying inequality (41) to $\nabla_{H} \beta$, also by (105) and (106), we reach

$$
\left\|\nabla_{H} \beta\right\|_{\infty} \leq C\left(\left\|\nabla_{H} \cdot \beta\right\|_{\infty}+\left\|\nabla_{H}^{\perp} \cdot \beta\right\|_{\infty}\right)\left(1+\log ^{+}\left\|\nabla_{H} \beta\right\|_{H^{2}(M)}\right) \leq C\|T\|_{\infty}\left(1+\log ^{+}\|T\|_{H^{2}(M)}\right)
$$

Therefore, by (115)-(118), we infer that

$$
\begin{aligned}
& \left\|\nabla_{H} v\right\|_{\infty} \leq C\left\|\nabla_{H} \bar{v}\right\|_{H^{1}(M)}\left(1+\log ^{+}\left\|\nabla_{H} \bar{v}\right\|_{H^{2}(M)}\right)^{1 / 2}+C \int_{-h}^{0}\left[\|T\|_{\infty}\left(1+\log ^{+}\|T\|_{H^{2}(M)}\right)\right] d z \\
& +C\left\|\int_{-h}^{0}\left|\nabla_{H} \zeta\right| d z\right\|_{H^{1}(M)}\left(1+\log ^{+}\left\|\int_{-h}^{0}\left|\nabla_{H} \zeta\right| d z\right\|_{H^{2}(M)}\right)^{1 / 2} \\
& \leq C\left\|\nabla_{H} \bar{v}\right\|_{H^{1}(M)}\left(1+\log ^{+}\left\|\nabla_{H} \bar{v}\right\|_{H^{2}(M)}\right)^{1 / 2}+C\|T\|_{\infty}\left(1+\log ^{+} \int_{-h}^{0}\|T\|_{H^{2}(M)} d z\right) \\
& +C\left\|\int_{-h}^{0}\left|\nabla_{H} \zeta\right| d z\right\|_{H^{1}(M)}\left(1+\log ^{+}\left\|\int_{-h}^{0}\left|\nabla_{H} \zeta\right| d z\right\|_{H^{2}(M)}\right)^{1 / 2} \\
& \leq C\left\|\nabla_{H} \bar{v}\right\|_{H^{1}(M)}\left(1+\log ^{+}\left\|\nabla_{H} \bar{v}\right\|_{H^{2}(M)}\right)^{1 / 2}+C\|T\|_{\infty}\left(1+\log ^{+}\left\|\Delta_{H} T\right\|_{L^{2}(\Omega)}\right) \\
& +C\left(\|\eta\|_{H^{1}(\Omega)}+\|\theta\|_{H^{1}(\Omega)}\right)\left[1+\log ^{+}\left(\left\|\Delta_{H} \eta\right\|_{L^{2}(\Omega)}+\left\|\Delta_{H} \theta\right\|_{L^{2}(\Omega)}\right)\right]^{1 / 2} .
\end{aligned}
$$

3.8.1. $\left\|\nabla_{H} \Delta_{H} \bar{v}\right\|_{2}^{2}$ estimates. By taking the $\Delta_{H}$ to equation (29) and then taking the inner product of equation (29) with $-\Delta_{H}^{2} \bar{v}$ in $L^{2}(M)$, we reach

$$
\begin{aligned}
& \frac{1}{2} \frac{d\left\|\nabla_{H} \Delta_{H} \bar{v}\right\|_{2}^{2}}{d t}+\frac{1}{R_{1}}\left\|\Delta_{H}^{2} \bar{v}\right\|_{2}^{2} \\
& =\int_{M} \Delta_{H}\left\{\left(\bar{v} \cdot \nabla_{H}\right) \bar{v}+\overline{\left[\left(\widetilde{v} \cdot \nabla_{H}\right) \widetilde{v}+\left(\nabla_{H} \cdot \widetilde{v}\right) \widetilde{v}\right.}+f_{0} \vec{k} \times \bar{v}\right\} \cdot \Delta_{H}^{2} \bar{v} d x d y .
\end{aligned}
$$


Integrating by parts and applying (30), we obtain

$$
\begin{aligned}
& \frac{1}{2} \frac{d\left\|\nabla_{H} \Delta_{H} \bar{v}\right\|_{2}^{2}}{d t}+\frac{1}{R_{1}}\left\|\Delta_{H}^{2} \bar{v}\right\|_{2}^{2} \\
& \leq C \int_{M}\left\{|\bar{v}|\left|\nabla_{H}^{3} \bar{v}\right|+\left|\nabla_{H} \bar{v}\right|\left|\nabla_{H}^{2} \bar{v}\right|+\int_{-h}^{0}\left(|\widetilde{v}|\left|\nabla_{H}^{3} \widetilde{v}\right|+\left|\nabla_{H} \widetilde{v}\right|\left|\nabla_{H}^{2} \widetilde{v}\right|\right) d z+\left|\Delta_{H} \bar{v}\right|\right\}\left|\Delta_{H}^{2} \bar{v}\right| d x d y \\
& \leq C \int_{M}\left\{|\bar{v}|\left|\nabla_{H}^{3} \bar{v}\right|+\left|\nabla_{H} \bar{v}\right|\left|\nabla_{H}^{2} \bar{v}\right|+\left(\int_{-h}^{0}|\widetilde{v}| d z\right)\left(\int_{-h}^{0}\left(\left|\nabla_{H}^{3} \zeta\right|+\left|\nabla_{H}^{3} \beta\right|\right) d z\right)\right. \\
& \left.+\left(\int_{-h}^{0}\left(\left|\nabla_{H} \zeta\right|+\left|\nabla_{H} \beta\right|\right) d z\right)\left(\int_{-h}^{0}\left(\left|\nabla_{H}^{2} \zeta\right|+\left|\nabla_{H}^{2} \beta\right|\right) d z\right)+\left|\Delta_{H} \bar{v}\right|\right\}\left|\Delta_{H}^{2} \bar{v}\right| d x d y .
\end{aligned}
$$

By applying Hölder inequality, (33), (36), 39, (44) and (110) to the above estimate, we obtain

$$
\begin{aligned}
& \quad \frac{1}{2} \frac{d\left\|\nabla_{H} \Delta_{H} \bar{v}\right\|_{2}^{2}}{d t}+\frac{1}{R_{1}}\left\|\Delta_{H}^{2} \bar{v}\right\|_{2}^{2} \\
& \leq C\left\{\|\bar{v}\|_{4}\left\|\nabla_{H}^{3} \bar{v}\right\|_{4}+\left\|\nabla_{H} \bar{v}\right\|_{4}\left\|\nabla_{H}^{2} \bar{v}\right\|_{4}+\int_{-h}^{0}\|\widetilde{v}\|_{\infty} d z\left\|\int_{-h}^{0}\left(\left|\nabla_{H}^{3} \zeta\right|+\left|\nabla_{H}^{3} \beta\right|\right) d z\right\|_{2}\right. \\
& \left.\quad+\left\|\int_{-h}^{0}\left(\left|\nabla_{H} \zeta\right|+\left|\nabla_{H} \beta\right|\right) d z\right\|_{4}\left\|\int_{-h}^{0}\left(\left|\nabla_{H}^{2} \zeta\right|+\left|\nabla_{H}^{2} \beta\right|\right) d z\right\|_{4}+\left\|\Delta_{H} \bar{v}\right\|_{2}\right\}\left\|\Delta_{H}^{2} \bar{v}\right\|_{2} \\
& \leq C \\
& \quad+\|\bar{v}\|_{2}^{\frac{1}{2}}\left\|\nabla_{H} \bar{v}\right\|_{2}^{\frac{1}{2}}\left\|\nabla_{H}^{3} \bar{v}\right\|_{2}^{\frac{1}{2}}\left\|\Delta_{H}^{2} \bar{v}\right\|_{2}^{\frac{1}{2}}+\left\|\nabla_{H} \bar{v}\right\|_{2}\left\|\nabla_{H}^{3} \bar{v}\right\|_{2} \\
& \quad+\|\widetilde{v}\|_{2}^{\frac{1}{2}}\left\|\nabla_{H} \widetilde{v}\right\|_{\infty}^{\frac{1}{2}}\left(\left\|\Delta_{H} \eta\right\|_{2}+\left\|\Delta_{H} \theta\right\|_{2}+\left\|\Delta_{H} T\right\|_{2}\right) \\
& \left.\quad+\left(\|\eta\|_{2}+\|\theta\|_{2}+\|T\|_{2}\right)\left(\left\|\nabla_{H}^{2} \eta\right\|_{2}+\left\|\nabla_{H}^{2} \theta\right\|_{2}+\left\|\nabla_{H}^{2} T\right\|_{2}\right)+\left\|\Delta_{H} \theta\right\|_{2}\right\}\left\|\Delta_{H}^{2} \bar{v}\right\|_{2} .
\end{aligned}
$$

Thus, by Young's and the Cauchy-Schwarz inequalities, we have

$$
\begin{aligned}
& \frac{d\left\|\nabla_{H} \Delta_{H} \bar{v}\right\|_{2}^{2}}{d t}+\frac{1}{R_{1}}\left\|\Delta_{H}^{2} \bar{v}\right\|_{2}^{2} \\
& \quad \leq C\left(\|\bar{v}\|_{2}^{2}\left\|\nabla_{H} \bar{v}\right\|_{2}^{2}+\left\|\nabla_{H} \bar{v}\right\|_{2}^{2}\right)\left\|\nabla_{H} \Delta_{H} \bar{v}\right\|_{2}^{2} \\
& \quad+\left(\|\widetilde{v}\|_{2}\left\|\nabla_{H} \widetilde{v}\right\|_{\infty}+\|\eta\|_{2}^{2}+\|\theta\|_{2}^{2}+\|T\|_{2}^{2}\right)\left(\left\|\Delta_{H} \eta\right\|_{2}^{2}+\left\|\Delta_{H} \theta\right\|_{2}^{2}+\left\|\Delta_{H} T\right\|_{2}^{2}\right)
\end{aligned}
$$


3.8.2. $\left\|\Delta_{H} T\right\|_{2}+\left\|\nabla_{H} T_{z}\right\|_{2}$ estimates. By applying the operator $\Delta_{H}$ to equation (24), and then taking the inner product of equation (24) with $\Delta_{H} T+T_{z z}$ in $L^{2}(\Omega)$, we get

$$
\begin{aligned}
\frac{1}{2} & \frac{d\left(\left\|\Delta_{H} T\right\|_{2}^{2}+\left\|\nabla_{H} T_{z}\right\|_{2}^{2}\right)}{d t}+\frac{1}{R_{3}}\left(\left\|\Delta_{H} T_{z}\right\|_{2}^{2}+\left\|\nabla_{H} T_{z z}\right\|_{2}^{2}\right) \\
= & -\int_{\Omega} \Delta_{H}\left[v \cdot \nabla_{H} T-\left(\int_{-h}^{z} \nabla_{H} \cdot v(x, y, \xi, t) d \xi\right)\left(\frac{\partial T}{\partial z}+\frac{1}{h}\right)-Q\right] \Delta_{H} T d x d y d z \\
& -\int_{\Omega} \nabla_{H}\left[v \cdot \nabla_{H} T_{z}-\left(\int_{-h}^{z} \nabla_{H} \cdot v(x, y, \xi, t) d \xi\right) \frac{\partial^{2} T}{\partial z^{2}}+u \cdot \nabla_{H} T\right. \\
& \left.-\left(\nabla_{H} \cdot v\right)\left(\frac{\partial T}{\partial z}+\frac{1}{h}\right)-Q_{z}\right] \cdot \nabla_{H} T_{z} d x d y d z \\
= & -\int_{\Omega}\left[\Delta_{H} v \cdot \nabla_{H} T+2 \nabla_{H} v \cdot \nabla_{H}^{2} T-\left(\int_{-h}^{z} \Delta_{H}\left(\nabla_{H} \cdot v(x, y, \xi, t)\right) d \xi\right)\left(\frac{\partial T}{\partial z}+\frac{1}{h}\right)\right. \\
& \left.-2\left(\int_{-h}^{z} \nabla_{H}\left(\nabla_{H} \cdot v(x, y, \xi, t)\right) d \xi\right) \nabla_{H} T_{z}-\Delta_{H} Q\right] \Delta_{H} T d x d y d z \\
& -\int_{\Omega}\left[\nabla_{H} v \cdot \nabla_{H} T_{z}-\left(\int_{-h}^{z} \nabla_{H}\left(\nabla_{H} \cdot v(x, y, \xi, t)\right) d \xi\right) \frac{\partial^{2} T}{\partial z^{2}}\right. \\
& \left.+\nabla_{H} u \cdot \nabla_{H} T+u \cdot \nabla_{H}^{2} T-\nabla_{H}\left(\nabla_{H} \cdot v\right)\left(\frac{\partial T}{\partial z}+\frac{1}{h}\right)-\left(\nabla_{H} \cdot v\right) \nabla_{H} T_{z}-\nabla_{H} Q_{z}\right] \cdot \nabla_{H} T_{z} d x d y d z \\
= & -\int_{\Omega}\left[\Delta_{H} v \cdot \nabla_{H} T+2 \nabla_{H} v \cdot \nabla_{H}^{2} T-\frac{1}{h}\left(\int_{-h}^{z} \Delta_{H}\left(\nabla_{H} \cdot v(x, y, \xi, t)\right) d \xi\right)+\Delta_{H}\left(\nabla_{H} \cdot v\right) T\right. \\
& \left.+2 \nabla_{H}\left(\nabla_{H} \cdot v\right) \cdot \nabla_{H} T-\Delta_{H} Q\right] \Delta_{H} T d x d y d z \\
& -\int_{\Omega}\left[\int_{-h}^{z} \Delta_{H}\left(\nabla_{H} \cdot v(x, y, \xi, t)\right) d \xi T+2 \int_{-h}^{z} \nabla_{H}\left(\nabla_{H} \cdot v(x, y, \xi, t)\right) d \xi \cdot \nabla_{H} T\right] \cdot \Delta_{H} T_{z} d x d y d z \\
& -\int_{\Omega}\left[\nabla_{H} v \cdot \nabla_{H} T_{z}+\left(\nabla_{H}\left(\nabla_{H} \cdot v\right)\right) \frac{\partial T}{\partial z}+\nabla_{H} u \cdot \nabla_{H} T+u \cdot \nabla_{H}^{2} T\right. \\
& \left.-\frac{1}{h} \nabla_{H}\left(\nabla_{H} \cdot v\right)+\nabla_{H}\left(\nabla_{H} \cdot u\right) T+\left(\nabla_{H} \cdot u\right) \nabla_{H} T-\nabla_{H} Q z\right] \cdot \nabla_{H} T_{z} d x d y d z \\
& -\int_{\Omega}\left[\left(\int_{-h}^{z} \nabla_{H}\left(\nabla_{H} \cdot v(x, y, \xi, t)\right) d \xi\right) \frac{\partial T}{\partial z}+\nabla_{H}\left(\nabla_{H} \cdot v\right) T+\left(\nabla_{H} \cdot v\right) \nabla_{H} T\right] \cdot \nabla_{H} T_{z z} d x d y d z .
\end{aligned}
$$


Thus,

$$
\begin{aligned}
\frac{1}{2} & \frac{d\left(\left\|\Delta_{H} T\right\|_{2}^{2}+\left\|\nabla_{H} T_{z}\right\|_{2}^{2}\right)}{d t}+\frac{1}{R_{3}}\left(\left\|\Delta_{H} T_{z}\right\|_{2}^{2}+\left\|\nabla_{H} T_{z z}\right\|_{2}^{2}\right) \\
\leq & C \int_{\Omega}\left\{\left|\nabla_{H} v\right|\left|\nabla_{H}^{2} T\right|+\left|\Delta_{H} v\right|\left|\nabla_{H} T\right|+\overline{\left|\Delta_{H}\left(\nabla_{H} \cdot v\right)\right|}+\left|\nabla_{H}^{2}\left(\nabla_{H} \cdot v\right)\right||T|+\left|\nabla_{H}\left(\nabla_{H} \cdot v\right)\right|\left|\nabla_{H} T\right|\right. \\
& \left.+\left|\Delta_{H} Q\right|\right\}\left|\Delta_{H} T\right| d x d y d z \\
& +C \int_{\Omega}\left\{\left(\int_{-h}^{0}\left|\Delta_{H}\left(\nabla_{H} \cdot v\right)\right| d z|T|+\int_{-h}^{0}\left|\nabla_{H}\left(\nabla_{H} \cdot v\right)\right| d z\left|\nabla_{H} T\right|\right)\left|\Delta_{H} T_{z}\right|\right\} d x d y d z \\
& +C \int_{\Omega}\left\{\left|\nabla_{H} v\right|\left|\nabla_{H} T_{z}\right|+\left|\nabla_{H}\left(\nabla_{H} \cdot v\right)\right|\left|T_{z}\right|+\left|\nabla_{H} u\right|\left|\nabla_{H} T\right|+|u|\left|\nabla_{H}^{2} T\right|\right. \\
& \left.+\left|\nabla_{H}\left(\nabla_{H} \cdot v\right)\right|+\left|\nabla_{H}\left(\nabla_{H} \cdot u\right)\right||T|+\left|\nabla_{H} Q_{z}\right|\right\}\left|\nabla_{H} T_{z}\right| d x d y d z \\
& +C \int_{\Omega}\left[\left(\int_{-h}^{0}\left|\nabla_{H}\left(\nabla_{H} \cdot v\right)\right| d z\right)\left|T_{z}\right|+\left|\nabla_{H}\left(\nabla_{H} \cdot v\right)\right||T|+\left|\nabla_{H} \cdot v\right|\left|\nabla_{H} T\right|\right]\left|\nabla_{H} T_{z z}\right| d x d y d z .
\end{aligned}
$$

Thanks to (107)-(109), we obtain

$$
\begin{aligned}
& \frac{1}{2} \frac{d\left(\left\|\Delta_{H} T\right\|_{2}^{2}+\left\|\nabla_{H} T_{z}\right\|_{2}^{2}\right)}{d t}+\frac{1}{R_{3}}\left(\left\|\Delta_{H} T_{z}\right\|_{2}^{2}+\left\|\nabla_{H} T_{z z}\right\|_{2}^{2}\right) \\
& \leq C \int_{\Omega}\left\{\left|\nabla_{H} v\right|\left|\nabla_{H}^{2} T\right|+\int_{-h}^{0}\left(\left|\Delta_{H} \zeta\right|+\left|\nabla_{H} T\right|\right) d z\left|\nabla_{H} T\right|+\int_{-h}^{0}\left(\left|\Delta_{H} \theta\right|+\left|\Delta_{H} T\right|\right) d z\right. \\
& \left.+\int_{-h}^{0}\left(\left|\nabla_{H}^{2} \theta\right|+\left|\nabla_{H}^{2} T\right|\right) d z|T|+\int_{-h}^{0}\left(\left|\nabla_{H} \theta\right|+\left|\nabla_{H} T\right|\right) d z\left|\nabla_{H} T\right|+\left|\Delta_{H} Q\right|\right\}\left|\Delta_{H} T\right| d x d y d z \\
& +C \int_{\Omega}\left\{\left(\int_{-h}^{0}\left(\left|\nabla_{H}^{2} \theta\right|+\left|\nabla_{H}^{2} T\right|\right) d z|T|+\int_{-h}^{0}\left(\left|\nabla_{H} \theta\right|+\left|\nabla_{H} T\right|\right) d z\left|\nabla_{H} T\right|\right)\left|\Delta_{H} T_{z}\right|\right\} d x d y d z \\
& +C \int_{\Omega}\left\{\left|\nabla_{H} v\right|\left|\nabla_{H} T_{z}\right|+\int_{-h}^{0}\left(\left|\nabla_{H} \theta\right|+\left|\nabla_{H} T\right|\right) d z\left|T_{z}\right|+\left|\nabla_{H} u\right|\left|\nabla_{H} T\right|\right. \\
& \left.+|u|\left|\nabla_{H}^{2} T\right|+\int_{-h}^{0}\left(\left|\nabla_{H} \theta\right|+\left|\nabla_{H} T\right|\right) d z+\left(\left|\nabla_{H} \theta\right|+\left|\nabla_{H} T\right|\right)|T|+\left|\nabla_{H} Q_{z}\right|\right\}\left|\nabla_{H} T_{z}\right| d x d y d z \\
& +C \int_{\Omega}\left[\left(\int_{-h}^{0}\left(\left|\nabla_{H} \theta\right|+\left|\nabla_{H} T\right|\right) d z\right)\left|T_{z}\right|+\left(\left|\nabla_{H} \theta\right|+\left|\nabla_{H} T\right|\right)|T|\right. \\
& \left.\quad+\int_{-h}^{0}(|\theta|+|T|) d z\left|\nabla_{H} T\right|\right]\left|\nabla_{H} T_{z z}\right| d x d y d z .
\end{aligned}
$$


Using Hölder inequality, and inequalities (44), (45) and (110), we obtain

$$
\begin{aligned}
& \frac{1}{2} \frac{d\left(\left\|\Delta_{H} T\right\|_{2}^{2}+\left\|\nabla_{H} T_{z}\right\|_{2}^{2}\right)}{d t}+\frac{1}{R_{3}}\left(\left\|\Delta_{H} T_{z}\right\|_{2}^{2}+\left\|\nabla_{H} T_{z z}\right\|_{2}^{2}\right) \\
& \leq C\left\|\nabla_{H} v\right\|_{\infty}\left\|\Delta_{H} T\right\|_{2}^{2}+C\left\|\Delta_{H} \zeta\right\|_{2}^{1 / 2}\left\|\nabla_{H} \Delta_{H} \zeta\right\|_{2}^{1 / 2}\|T\|_{\infty}^{1 / 2}\left\|\nabla_{H}^{2} T\right\|_{2}^{3 / 2}+C\|T\|_{\infty}\left\|\nabla_{H}^{2} T\right\|_{2}^{2} \\
& +C\left\|\nabla_{H}^{2} \theta\right\|_{2}\left(1+\|T\|_{\infty}\right)\left\|\Delta_{H} T\right\|_{2}+C\left\|\nabla_{H} \theta\right\|_{2}^{1 / 2}\left\|\nabla_{H}^{2} \theta\right\|_{2}^{1 / 2}\|T\|_{\infty}^{1 / 2}\left\|\nabla_{H}^{2} T\right\|_{2}^{3 / 2}+C\left\|\Delta_{H} Q\right\|_{2}\left\|\Delta_{H} T\right\|_{2} \\
& +C\left(\left\|\nabla_{H}^{2} \theta\right\|_{2}+\left\|\nabla_{H}^{2} T\right\|_{2}\right)\|T\|_{\infty}\left\|\Delta_{H} T_{z}\right\|_{2}+C\left\|\nabla_{H} \theta\right\|_{2}^{1 / 2}\left\|\nabla_{H}^{2} \theta\right\|_{2}^{1 / 2}\|T\|_{\infty}^{1 / 2}\left\|\nabla_{H}^{2} T\right\|_{2}^{1 / 2}\left\|\Delta_{H} T_{z}\right\|_{2} \\
& +C\left\|\nabla_{H} v\right\|_{\infty}\left\|\nabla_{H} T_{z}\right\|_{2}^{2}+C\left\|\nabla_{H} \theta\right\|_{2}^{1 / 2}\left\|\nabla_{H}^{2} \theta\right\|_{2}^{1 / 2}\left\|T_{z}\right\|_{2}^{1 / 2}\left\|\nabla_{H} T_{z}\right\|_{2}^{3 / 2} \\
& +C\|T\|_{\infty}^{1 / 2}\left\|\nabla_{H}^{2} T\right\|_{2}^{1 / 2}\left\|T_{z}\right\|_{2}^{1 / 2}\left\|\nabla_{H} T_{z}\right\|_{2}^{3 / 2}+C\left\|\nabla_{H} u\right\|_{3}\left\|\nabla_{H} T\right\|_{6}\left\|\nabla_{H} T_{z}\right\|_{2}+C\|u\|_{\infty}\left\|\nabla_{H}^{2} T\right\|_{2}\left\|\nabla_{H} T_{z}\right\|_{2} \\
& +C\left(\left\|\nabla_{H} \theta\right\|_{2}+\left\|\nabla_{H} T\right\|_{2}\right)\left(1+\|T\|_{\infty}\right) \\
& +C\left(\left\|\nabla_{H} \theta\right\|_{2}^{1 / 2}\left\|\nabla_{H}^{2} \theta\right\|_{2}^{1 / 2}+C\|T\|_{\infty}^{1 / 2}\left\|\nabla_{H}^{2} T\right\|_{2}^{1 / 2}\right)\left\|T_{z}\right\|_{2}^{1 / 2}\left\|\nabla_{H} T_{z}\right\|_{2}^{1 / 2}\left\|\nabla_{H} T_{z z}\right\|_{2} \\
& +\left\|\nabla_{H} Q_{z}\right\|_{2}\left\|\nabla_{H} T_{z}\right\|_{2}+C\left(\left\|\nabla_{H} \theta\right\|_{2}+\left\|\nabla_{H} T\right\|_{2}\right)\|T\|_{\infty}\left\|\nabla_{H} T_{z z}\right\|_{2} \\
& +C\|\theta\|_{2}^{1 / 2}\left\|\nabla_{H} \theta\right\|_{2}^{1 / 2}\|T\|_{\infty}^{1 / 2}\left\|\nabla_{H}^{2} T\right\|_{2}^{1 / 2}\left\|\nabla_{H} T_{z z}\right\|_{2} \\
& \leq C\left\|\nabla_{H} v\right\|_{\infty}\left(\left\|\Delta_{H} T\right\|_{2}^{2}+\left\|\nabla_{H} T_{z}\right\|_{2}^{2}\right)+C\left(\left\|\nabla_{H} \eta\right\|_{2}+\left\|\nabla_{H} \theta\right\|_{2}\right)^{1 / 2}\left(\left\|\Delta_{H} \eta\right\|_{2}+\left\|\Delta_{H} \theta\right\|_{2}\right)^{1 / 2}\|T\|_{\infty}^{1 / 2}\left\|\nabla_{H}^{2} T\right\|_{2}^{3 / 2} \\
& +C\left(\left\|\Delta_{H} \eta\right\|_{2}+\left\|\Delta_{H} \theta\right\|_{2}+\left\|\Delta_{H} T\right\|_{2}\right)\left(1+\|T\|_{\infty}\right)\left\|\Delta_{H} T\right\|_{2}+C\left\|\Delta_{H} Q\right\|_{2}\left\|\Delta_{H} T\right\|_{2}+C\left\|\nabla_{H} Q_{z}\right\|_{2}\left\|\nabla_{H} T_{z}\right\|_{2} \\
& +\left(\left\|\nabla_{H} \eta\right\|_{2}^{1 / 2}\left\|\nabla_{H}^{2} \eta\right\|_{2}^{\frac{1}{2}}+\left\|\nabla_{H} \theta\right\|_{2}^{\frac{1}{2}}\left\|\nabla_{H}^{2} \theta\right\|_{2}^{\frac{1}{2}}+\|T\|_{\infty}^{\frac{1}{2}}\left\|\Delta_{H} T\right\|_{2}^{\frac{1}{2}}\right)\|T\|_{\infty}^{\frac{1}{2}}\left\|\Delta_{H} T\right\|_{2}^{\frac{1}{2}}\left(\left\|\Delta_{H} T\right\|_{2}+\left\|\Delta_{H} T_{z}\right\|_{2}\right) \\
& +C\|u\|_{6}\left\|\nabla_{H} T_{z}\right\|_{2}^{\frac{1}{2}}\left\|\nabla_{H}^{2} T_{z}\right\|_{2}^{\frac{1}{2}}\left\|\Delta_{H} T\right\|+C\left[\left\|\nabla_{H} \theta\right\|_{2}^{\frac{1}{2}}\left\|\nabla_{H}^{2} \theta\right\|_{2}^{\frac{1}{2}}\left\|T_{z}\right\|_{2}^{\frac{1}{2}}\left\|\nabla_{H} T_{z}\right\|_{2}^{\frac{1}{2}}\right. \\
& \left.+\left(\|T\|_{4}+\|\theta\|_{2}^{1 / 2}\left\|\nabla_{H} \theta\right\|_{2}^{1 / 2}+\left\|T_{z}\right\|_{2}^{1 / 2}\left\|\nabla_{H} T_{z}\right\|_{2}^{1 / 2}\right)\|T\|_{\infty}^{1 / 2}\left\|\Delta_{H} T\right\|_{2}^{1 / 2}\right]\left\|\nabla_{H} T_{z z}\right\|_{2} .
\end{aligned}
$$

By the Cauchy-Schwarz and Young's inequalities, we reach

$$
\begin{aligned}
& \frac{d\left(\left\|\Delta_{H} T\right\|_{2}^{2}+\left\|\nabla_{H} T_{z}\right\|_{2}^{2}\right)}{d t}+\frac{1}{R_{3}}\left(\left\|\Delta_{H} T_{z}\right\|_{2}^{2}+\left\|\nabla_{H} T_{z z}\right\|_{2}^{2}\right) \\
& \leq C\left\|\nabla_{H} v\right\|_{\infty}\left(\left\|\Delta_{H} T\right\|_{2}^{2}+\left\|\nabla_{H} T_{z}\right\|_{2}^{2}\right)+C\left(\left\|\nabla_{H} \eta\right\|_{2}+\left\|\nabla_{H} \theta\right\|_{2}\right)^{1 / 2}\left(\left\|\Delta_{H} \eta\right\|_{2}+\left\|\Delta_{H} \theta\right\|_{2}\right)^{1 / 2}\|T\|_{\infty}^{1 / 2}\left\|\Delta_{H} T\right\|_{2}^{3 / 2} \\
& \quad+C\left(\left\|\Delta_{H} \eta\right\|_{2}+\left\|\Delta_{H} \theta\right\|_{2}+\left\|\Delta_{H} T\right\|_{2}\right)\|T\|_{\infty}\left\|\Delta_{H} T\right\|_{2}+C\left\|\Delta_{H} Q\right\|_{2}\left\|\Delta_{H} T\right\|_{2}+C\left\|\nabla_{H} Q_{z}\right\|_{2}\left\|\nabla_{H} T_{z}\right\|_{2} \\
& \quad+\left(\left\|\nabla_{H} \eta\right\|_{2}^{1 / 2}\left\|\Delta_{H} \eta\right\|_{2}^{\frac{1}{2}}+\left\|\nabla_{H} \theta\right\|_{2}^{\frac{1}{2}}\left\|\Delta_{H} \theta\right\|_{2}^{\frac{1}{2}}+\|T\|_{\infty}^{\frac{1}{2}}\left\|\Delta_{H} T\right\|_{2}^{\frac{1}{2}}\right)\|T\|_{\infty}^{\frac{1}{2}}\left\|\Delta_{H} T\right\|_{2}^{\frac{1}{2}}\left\|\Delta_{H} T\right\|_{2} \\
& \quad+\left(\left\|\nabla_{H} \eta\right\|_{2}\left\|\Delta_{H} \eta\right\|_{2}+\left\|\nabla_{H} \theta\right\|_{2}\left\|\Delta_{H} \theta\right\|_{2}+\|T\|_{\infty}\left\|\Delta_{H} T\right\|_{2}\right)\|T\|_{\infty}\left\|\Delta_{H} T\right\|_{2}+C\|u\|_{6}^{3}\left(\left\|\nabla_{H} T_{z}\right\|_{2}^{2}+\left\|\Delta_{H} T\right\|^{2}\right) \\
& \quad+C\left[\left\|\nabla_{H} \theta\right\|_{2}\left\|\Delta_{H} \theta\right\|_{2}\left\|T_{z}\right\|_{2}\left\|\nabla_{H} T_{z}\right\|_{2}+\left(\|T\|_{4}^{2}+\|\theta\|_{2}\left\|\nabla_{H} \theta\right\|_{2}+\left\|T_{z}\right\|_{2}\left\|\nabla_{H} T_{z}\right\|_{2}\right)\|T\|_{\infty}\left\|\Delta_{H} T\right\|_{2}\right] \\
& \leq C\left\|\Delta_{H} Q\right\|_{2}^{2}+C\left\|\nabla_{H} Q_{z}\right\|_{2}^{2}+C\|T\|_{\infty}^{4}+C\left\|\nabla_{H} v\right\|_{\infty}\left(\left\|\Delta_{H} T\right\|_{2}^{2}+\left\|\nabla_{H} T_{z}\right\|_{2}^{2}\right) \\
& \quad+C\left(1+\left\|\nabla_{H} \eta\right\|_{2}^{2}+\left\|\nabla_{H} \theta\right\|_{2}^{2}+\|T\|_{\infty}^{2}+\|u\|_{6}^{3}+\left\|T_{z}\right\|_{2}^{2}\right)\left(\left\|\Delta_{H} T\right\|_{2}^{2}+\left\|\nabla_{H} T_{z}\right\|_{2}^{2}+\left\|\Delta_{H} \eta\right\|_{2}^{2}+\left\|\Delta_{H} \theta\right\|_{2}^{2}\right) .
\end{aligned}
$$

Thus, we get

$$
\begin{aligned}
& \frac{d\left(\left\|\Delta_{H} T\right\|_{2}^{2}+\left\|\nabla_{H} T_{z}\right\|_{2}^{2}\right)}{d t}+\frac{1}{R_{3}}\left(\left\|\Delta_{H} T_{z}\right\|_{2}^{2}+\left\|\nabla_{H} T_{z z}\right\|_{2}^{2}\right) \\
& \leq C\left\|\Delta_{H} Q\right\|_{2}^{2}+C\left\|\nabla_{H} Q_{z}\right\|_{2}^{2}+C\|T\|_{\infty}^{4}+C\left\|\nabla_{H} v\right\|_{\infty}\left(\left\|\Delta_{H} T\right\|_{2}^{2}+\left\|\nabla_{H} T_{z}\right\|_{2}^{2}\right) \\
& \quad+C\left(1+\left\|\nabla_{H} \eta\right\|_{2}^{2}+\left\|\nabla_{H} \theta\right\|_{2}^{2}+\|T\|_{\infty}^{2}+\|u\|_{6}^{3}+\left\|T_{z}\right\|_{2}^{2}\right)\left(\left\|\Delta_{H} T\right\|_{2}^{2}+\left\|\nabla_{H} T_{z}\right\|_{2}^{2}+\left\|\Delta_{H} \eta\right\|_{2}^{2}+\left\|\Delta_{H} \theta\right\|_{2}^{2}\right) .
\end{aligned}
$$

3.8.3. $\left\|\nabla_{H}\left(\nabla_{H}^{\perp} \cdot v_{z}\right)\right\|_{H^{1}(\Omega)}^{2}+\left\|\nabla_{H}\left(\nabla_{H} \cdot v_{z}+R_{1} T\right)\right\|_{H^{1}(\Omega)}^{2}$ estimates. By acting with $\Delta_{H}$ on equation (111) and equation (112), then taking the inner product of equation (111) with $\Delta_{H} \eta+\eta_{z z}$ in $L^{2}$ and equation (112) with 
$\Delta_{H} \theta+\theta_{z z}$ in $L^{2}$, respectively, we get

$$
\begin{aligned}
& \frac{1}{2} \frac{d\left(\left\|\Delta_{H} \eta\right\|_{2}^{2}+\left\|\nabla_{H} \eta_{z}\right\|_{2}^{2}+\left\|\Delta_{H} \theta\right\|_{2}^{2}+\left\|\nabla_{H} \theta_{z}\right\|_{2}^{2}\right)}{d t} \\
& +\frac{1}{R_{1}}\left(\left\|\nabla_{H} \Delta_{H} \eta\right\|_{2}^{2}+\left\|\nabla_{H} \Delta_{H} \eta_{z}\right\|_{2}^{2}+\left\|\nabla_{H} \Delta_{H} \theta\right\|_{2}^{2}+\left\|\Delta_{H} \theta_{z}\right\|_{2}^{2}\right) \\
& +\frac{1}{R_{2}}\left(\left\|\Delta_{H} \eta_{z}\right\|_{2}^{2}+\left\|\nabla_{H} \eta_{z z}\right\|_{2}^{2}+\left\|\Delta_{H} \theta_{z}\right\|_{2}^{2}+\left\|\nabla_{H} \theta_{z z}\right\|_{2}^{2}\right) \\
& =\int_{\Omega} \nabla_{H}\left\{\nabla _ { H } ^ { \perp } \cdot \left[\left(v \cdot \nabla_{H}\right) u-\left(\int_{-h}^{z} \nabla_{H} \cdot v(x, y, \xi, t) d \xi\right) \frac{\partial u}{\partial z}\right.\right. \\
& \left.\left.+\left(u \cdot \nabla_{H}\right) v-\left(\nabla_{H} \cdot v\right) u\right]+f_{0}\left(R_{1} T-\theta\right)\right\} \cdot \nabla_{H}\left(\Delta_{H} \eta+\eta_{z z}\right) d x d y d z \\
& +\int_{\Omega} \nabla_{H}\left\{\nabla _ { H } \cdot \left[\left(v \cdot \nabla_{H}\right) u-\left(\int_{-h}^{z} \nabla_{H} \cdot v(x, y, \xi, t) d \xi\right) \frac{\partial u}{\partial z}\right.\right. \\
& \left.\left.+\left(u \cdot \nabla_{H}\right) v-\left(\nabla_{H} \cdot v\right) u\right]+f_{0} \eta\right\} \cdot \nabla_{H}\left(\Delta_{H} \theta+\theta_{z z}\right) d x d y d z \\
& -\int_{\Omega}\left\{R _ { 1 } \left[\nabla_{H} v \cdot \nabla_{H} T+v \cdot \nabla_{H}^{2} T-\left(\int_{-h}^{z} \nabla_{H}\left(\nabla_{H} \cdot v\right)(x, y, \xi, t) d \xi\right)\left(\frac{\partial T}{\partial z}+\frac{1}{h}\right)\right.\right. \\
& \left.\left.-\nabla_{H} Q+\left(\frac{1}{R_{3}}-\frac{1}{R_{2}}\right) \nabla_{H} T_{z z}\right]\right\} \cdot \nabla_{H}\left(\Delta_{H} \theta+\theta_{z z}\right) d x d y d z \\
& \left.-\int_{\Omega}\left[\left(\int_{-h}^{z} \nabla_{H}\left(\nabla_{H} \cdot v\right)(x, y, \xi, t) d \xi\right) \nabla_{H} T_{z} \Delta_{H} \theta+\left(\int_{-h}^{z} \nabla_{H} \cdot v(x, y, \xi, t) d \xi\right) \nabla_{H} T_{z} \cdot \nabla_{H} \theta_{z}\right)\right] d x d y d z \\
& \leq C \int_{\Omega}\left\{\left(|u|\left|\nabla_{H}^{3} v\right|+\left|\nabla_{H} u\right|\left|\nabla_{H}^{2} v\right|+\left|\nabla_{H}^{2} u\right|\left|\nabla_{H} v\right|+\left|\nabla_{H}^{3} u\right||v|+\left|u_{z}\right| \int_{-h}^{0}\left|\nabla_{H}^{2}\left(\nabla_{H} \cdot v\right)\right| d z\right.\right. \\
& \left.+\left|\nabla_{H} u_{z}\right| \int_{-h}^{0}\left|\nabla_{H}\left(\nabla_{H} \cdot v\right)\right| d z+\left|\nabla_{H}^{2} u_{z}\right| \int_{-h}^{0}\left|\nabla_{H} \cdot v\right| d z\right)\left(\left|\nabla_{H} \Delta_{H} \eta\right|+\left|\nabla_{H} \eta_{z z}\right|+\left|\nabla_{H} \Delta_{H} \theta\right|+\left|\nabla_{H} \theta_{z z}\right|\right) \\
& +C\left[\left|\nabla_{H} v\right|\left|\nabla_{H} T\right|+|v|\left|\nabla_{H}^{2} T\right|+\left(1+\left|T_{z}\right|\right) \int_{-h}^{0}\left|\nabla_{H}\left(\nabla_{H} \cdot v\right)\right| d z+\left|\nabla_{H} T_{z}\right| \int_{-h}^{0}\left|\nabla_{H} \cdot v\right| d z\right. \\
& \left.\left.+\left|\nabla_{H} Q\right|+\left|\frac{R_{1}}{R_{3}}-\frac{R_{1}}{R_{2}}\right|\left|\nabla_{H} T_{z z}\right|\right]\left(\left|\nabla_{H} \Delta_{H} \theta\right|+\left|\nabla_{H} \theta_{z z}\right|\right)\right\} d x d y d z+C\left(\left\|\Delta_{H} T\right\|_{2}^{2}+\left\|\Delta_{H} \eta\right\|_{2}^{2}+\left\|\Delta_{H} \theta\right\|_{2}^{2}\right) \\
& \leq C \int_{\Omega}\left\{\left[|u| \int_{-h}^{0}\left(\left|\nabla_{H}^{3} \zeta\right|+\left|\nabla_{H}^{3} \beta\right|\right) d z+\left(\left|\nabla_{H} \zeta\right|+\left|\nabla_{H} \beta\right|\right) \int_{-h}^{0}\left(\left|\nabla_{H}^{2} \zeta\right|+\left|\nabla_{H}^{2} \beta\right|\right) d z+\left(\left|\nabla_{H}^{3} \zeta\right|+\left|\nabla_{H}^{3} \beta\right|\right)|v|\right.\right. \\
& +\left(\left|\nabla_{H}^{2} \zeta\right|+\left|\nabla_{H}^{2} \beta\right|\right) \int_{-h}^{0}\left(\left|\nabla_{H} \zeta\right|+\left|\nabla_{H} \beta\right|\right) d z+\left|u_{z}\right| \int_{-h}^{0}\left(\left|\nabla_{H}^{2} \theta\right|+\left|\nabla_{H}^{2} T\right|\right) d z \\
& +\left(\left|\nabla_{H} \zeta_{z}\right|+\left|\nabla_{H} \beta_{z}\right|\right) \int_{-h}^{0}\left(\left|\nabla_{H} \theta\right|+\left|\nabla_{H} T\right|\right) d z \\
& \left.+\left(\left|\nabla_{H}^{2} \zeta_{z}\right|+\left|\nabla_{H}^{2} \beta_{z}\right|\right) \int_{-h}^{0}(|\theta|+|T|) d z\right]\left(\left|\nabla_{H} \Delta_{H} \eta\right|+\left|\nabla_{H} \eta_{z z}\right|+\left|\nabla_{H} \Delta_{H} \theta\right|+\left|\nabla_{H} \theta_{z z}\right|\right) \\
& +C\left[\left|\nabla_{H} T\right| \int_{-h}^{0}\left(\left|\nabla_{H} \zeta\right|+\left|\nabla_{H} \beta\right|\right) d z+|v|\left|\nabla_{H}^{2} T\right|\right. \\
& +\left(1+\left|T_{z}\right|\right) \int_{-h}^{0}\left(\left|\nabla_{H} \theta\right|+\left|\nabla_{H} T\right|\right) d z+\left|\nabla_{H} T_{z}\right| \int_{-h}^{0}(|\theta|+|T|) d z \\
& \left.\left.+\left|\nabla_{H} Q\right|+\left|\frac{R_{1}}{R_{3}}-\frac{R_{1}}{R_{2}}\right|\left|\nabla_{H} T_{z z}\right|\right]\left(\left|\nabla_{H} \Delta_{H} \theta\right|+\left|\nabla_{H} \theta_{z z}\right|\right)\right\} d x d y d z+C\left(\left\|\Delta_{H} T\right\|_{2}^{2}+\left\|\Delta_{H} \eta\right\|_{2}^{2}+\left\|\Delta_{H} \theta\right\|_{2}^{2}\right) .
\end{aligned}
$$


Using Hölder inequality, and inequalities (44), (45) and (110), we obtain

$$
\begin{aligned}
& \frac{1}{2} \frac{d\left(\left\|\Delta_{H} \eta\right\|_{2}^{2}+\left\|\nabla_{H} \eta_{z}\right\|_{2}^{2}+\left\|\Delta_{H} \theta\right\|_{2}^{2}+\left\|\nabla_{H} \theta_{z}\right\|_{2}^{2}\right)}{d t} \\
& +\frac{1}{R_{1}}\left(\left\|\nabla_{H} \Delta_{H} \eta\right\|_{2}^{2}+\left\|\nabla_{H} \Delta_{H} \eta_{z}\right\|_{2}^{2}+\left\|\nabla_{H} \Delta_{H} \theta\right\|_{2}^{2}+\left\|\Delta_{H} \theta_{z}\right\|_{2}^{2}\right) \\
& +\frac{1}{R_{2}}\left(\left\|\Delta_{H} \eta_{z}\right\|_{2}^{2}+\left\|\nabla_{H} \eta_{z z}\right\|_{2}^{2}+\left\|\Delta_{H} \theta_{z}\right\|_{2}^{2}+\left\|\nabla_{H} \theta_{z z}\right\|_{2}^{2}\right) \\
& \leq C\left(\left\|\nabla_{H} \Delta_{H} \eta\right\|_{2}+\left\|\nabla_{H} \eta_{z z}\right\|_{2}+\left\|\nabla_{H} \Delta_{H} \theta\right\|_{2}+\left\|\nabla_{H} \theta_{z z}\right\|_{2}\right)\left[\left(\|u\|_{\infty}+\|v\|_{\infty}\right)\left(\left\|\nabla_{H}^{2} \eta\right\|_{2}+\left\|\nabla_{H}^{2} \theta\right\|_{2}+\left\|\nabla_{H}^{2} T\right\|_{2}\right)\right. \\
& +\left(\|\eta\|_{2}^{\frac{1}{2}}\left\|\nabla_{H} \eta\right\|_{2}^{\frac{1}{2}}+\|\theta\|_{2}^{\frac{1}{2}}\left\|\nabla_{H} \theta\right\|_{2}^{\frac{1}{2}}+\|T\|_{\infty}\right)\left(\left\|\nabla_{H} \eta\right\|_{2}^{\frac{1}{2}}\left\|\nabla_{H}^{2} \eta\right\|_{2}^{\frac{1}{2}}+\left\|\nabla_{H} \theta\right\|_{2}^{\frac{1}{2}}\left\|\nabla_{H}^{2} \theta\right\|_{2}^{\frac{1}{2}}+\|T\|_{\infty}^{\frac{1}{2}}\left\|\nabla_{H}^{2} T\right\|_{2}^{\frac{1}{2}}\right) \\
& +\left(\left\|\nabla_{H} \eta\right\|_{2}^{\frac{1}{2}}\left\|\nabla_{H}^{2} \eta\right\|_{2}^{\frac{1}{2}}+\left\|\nabla_{H} \theta\right\|_{2}^{\frac{1}{2}}\left\|\nabla_{H}^{2} \theta\right\|_{2}^{\frac{1}{2}}+\|T\|_{\infty}^{\frac{1}{2}}\left\|\nabla_{H}^{2} T\right\|_{2}^{\frac{1}{2}}\right)\left(\|\eta\|_{2}^{\frac{1}{2}}\left\|\nabla_{H} \eta\right\|_{2}^{\frac{1}{2}}+\|\theta\|_{2}^{\frac{1}{2}}\left\|\nabla_{H} \theta\right\|_{2}^{\frac{1}{2}}+\|T\|_{\infty}\right) \\
& +\left(\left\|\theta_{z}\right\|_{2}^{\frac{1}{2}}\left\|\nabla_{H} \theta_{z}\right\|_{2}^{\frac{1}{2}}+\left\|T_{z}\right\|_{2}^{\frac{1}{2}}\left\|\nabla_{H} T_{z}\right\|_{2}^{\frac{1}{2}}\right)\left(\left\|\nabla_{H} \theta\right\|_{2}^{\frac{1}{2}}\left\|\nabla_{H}^{2} \theta\right\|_{2}^{\frac{1}{2}}+\|T\|_{\infty}^{\frac{1}{2}}\left\|\nabla_{H}^{2} T\right\|_{2}^{\frac{1}{2}}\right) \\
& +\left\|u_{z}\right\|_{2}^{\frac{1}{2}}\left\|\nabla_{H} u_{z}\right\|_{2}^{\frac{1}{2}}\left(\left\|\nabla_{H} \theta\right\|_{2}^{\frac{1}{2}}\left\|\nabla_{H}^{2} \theta\right\|_{2}^{\frac{1}{2}}+\|T\|_{\infty}^{\frac{1}{2}}\left\|\nabla_{H}^{2} T\right\|_{2}^{\frac{1}{2}}\right) \\
& \left.+\left(\left\|\nabla_{H} \theta_{z}\right\|_{2}^{\frac{1}{2}}\left\|\nabla_{H}^{2} \theta_{z}\right\|_{2}^{\frac{1}{2}}+\left\|\nabla_{H} T_{z}\right\|_{2}^{\frac{1}{2}}\left\|\nabla_{H}^{2} T_{z}\right\|_{2}^{\frac{1}{2}}\right)\left(\|\theta\|_{2}^{\frac{1}{2}}\left\|\nabla_{H} \theta\right\|_{2}^{\frac{1}{2}}+\|T\|_{\infty}\right)\right] \\
& +C\left[\left(\|\eta\|_{2}^{\frac{1}{2}}\left\|\nabla_{H} \eta\right\|_{2}^{\frac{1}{2}}+\|\theta\|_{2}^{\frac{1}{2}}\left\|\nabla_{H} \theta\right\|_{2}^{\frac{1}{2}}+\|T\|_{4}\right)\|T\|_{\infty}^{\frac{1}{2}}\left\|\Delta_{H} T\right\|_{2}^{\frac{1}{2}}+\|v\|_{\infty}\left\|\Delta_{H} T\right\|_{2}\right. \\
& +\left(\left\|\nabla_{H} \theta\right\|_{2}^{\frac{1}{2}}\left\|\nabla_{H}^{2} \theta\right\|_{2}^{\frac{1}{2}}+\|T\|_{\infty}^{\frac{1}{2}}\left\|\Delta_{H} T\right\|_{2}^{\frac{1}{2}}\right)\left\|T_{z}\right\|_{2}^{\frac{1}{2}}\left\|\nabla_{H} T_{z}\right\|_{2}^{\frac{1}{2}} \\
& +\left(\|\theta\|_{2}^{\frac{1}{2}}\left\|\nabla_{H} \theta\right\|_{2}^{\frac{1}{2}}+\|T\|_{\infty}\right)\left\|\nabla_{H} T_{z}\right\|_{2}^{\frac{1}{2}}\left\|\Delta_{H} T_{z}\right\|_{2}^{\frac{1}{2}}+\left\|\nabla_{H} \theta\right\|_{2}+\left\|\nabla_{H} T\right\|_{2}+\left\|\nabla_{H} Q\right\|_{2} \\
& \left.+\left|\frac{R_{1}}{R_{3}}-\frac{R_{1}}{R_{2}}\right|\left\|\nabla_{H} T_{z z}\right\|_{2}\right]\left(\left\|\nabla_{H} \Delta_{H} \theta\right\|_{2}+\left\|\nabla_{H} \theta_{z z}\right\|_{2}\right)+C\left(\left\|\Delta_{H} T\right\|_{2}^{2}+\left\|\Delta_{H} \eta\right\|_{2}^{2}+\left\|\Delta_{H} \theta\right\|_{2}^{2}\right)
\end{aligned}
$$

By Young's inequality and Cauchy-Schwarz inequality, we have

$$
\begin{aligned}
& \frac{d\left(\left\|\Delta_{H} \eta\right\|_{2}^{2}+\left\|\nabla_{H} \eta_{z}\right\|_{2}^{2}+\left\|\Delta_{H} \theta\right\|_{2}^{2}+\left\|\nabla_{H} \theta_{z}\right\|_{2}^{2}\right)}{d t}+\frac{1}{R_{1}}\left(\left\|\nabla_{H} \Delta_{H} \eta\right\|_{2}^{2}+\left\|\nabla_{H} \Delta_{H} \eta_{z}\right\|_{2}^{2}+\left\|\nabla_{H} \Delta_{H} \theta\right\|_{2}^{2}+\left\|\Delta_{H} \theta_{z}\right\|_{2}^{2}\right) \\
& \quad+\frac{1}{R_{2}}\left(\left\|\Delta_{H} \eta_{z}\right\|_{2}^{2}+\left\|\nabla_{H} \eta_{z z}\right\|_{2}^{2}+\left\|\Delta_{H} \theta_{z}\right\|_{2}^{2}+\left\|\nabla_{H} \theta_{z z}\right\|_{2}^{2}\right) \\
& \leq C\left(\left\|\Delta_{H} T\right\|_{2}^{2}+\left\|\nabla_{H} T_{z}\right\|_{2}^{2}+\left\|\Delta_{H} \eta\right\|_{2}^{2}+\left\|\nabla_{H} \eta_{z}\right\|_{2}^{2}+\left\|\Delta_{H} \theta\right\|_{2}^{2}+\left\|\nabla_{H} \theta_{z}\right\|_{2}^{2}\right)\left[1+\|T\|_{\infty}^{4}+\left\|\nabla_{H} u_{z}\right\|_{2}^{2}+\left\|u_{z z}\right\|_{2}^{2}\right. \\
& \left.\quad+\left\|\nabla_{H} \eta\right\|_{2}^{2}+\left\|\eta_{z}\right\|_{2}^{2}+\left\|\nabla_{H} \theta\right\|_{2}^{2}+\left\|\theta_{z}\right\|_{2}^{2}+\left\|T_{z}\right\|_{2}^{2}+\|\eta\|_{2}^{2}\left\|\nabla_{H} \eta\right\|_{2}^{2}+\|\theta\|_{2}^{2}\left\|\nabla_{H} \theta\right\|_{2}^{2}\right] \\
& \quad+\|\eta\|_{2}^{2}\left\|\nabla_{H} \eta\right\|_{2}^{2}+\|\theta\|_{2}^{2}\left\|\nabla_{H} \theta\right\|_{2}^{2}+\|T\|_{\infty}^{4}+\left\|u_{z}\right\|_{2}^{2}\left\|\nabla_{H} u_{z}\right\|_{2}^{2}+\left\|\nabla_{H} Q\right\|_{2}^{2} \\
& \quad+\frac{R_{1}^{2}\left(R_{1}+R_{2}\right)\left(R_{2}-R_{3}\right)^{2}}{R_{2}^{2} R_{3}^{2}}\left\|\nabla_{H} T_{z z}\right\|_{2}^{2} .
\end{aligned}
$$

Next, we will obtain an estimate for

$$
\left\|\nabla_{H} \Delta_{H} \bar{v}\right\|_{2}^{2}+\left\|\nabla_{H}\left(\nabla_{H}^{\perp} \cdot v_{z}\right)\right\|_{H^{1}(\Omega)}^{2}+\left\|\nabla_{H}\left(\nabla_{H} \cdot v_{z}+R_{1} T\right)\right\|_{H^{1}(\Omega)}^{2}+C_{R}\left\|\nabla_{H} T\right\|_{H^{1}(\Omega)}^{2},
$$

where $C_{R}=\frac{2 R_{1}^{2}\left(R_{1}+R_{2}\right)\left(R_{2}-R_{3}\right)^{2}}{R_{2}^{2} R_{3}}$. Denote by

$$
\begin{aligned}
& \mathcal{X}=1+\left\|\nabla_{H} \Delta_{H} \bar{v}\right\|_{2}^{2}+C_{R}\left\|\Delta_{H} T\right\|_{2}^{2}+C_{R}\left\|\nabla_{H} T_{z}\right\|_{2}^{2}+\left\|\Delta_{H} \eta\right\|_{2}^{2}+\left\|\nabla_{H} \eta_{z}\right\|_{2}^{2}+\left\|\Delta_{H} \theta\right\|_{2}^{2}+\left\|\nabla_{H} \theta_{z}\right\|_{2}^{2}, \\
& \mathcal{Y}=\left\|\Delta_{H}^{2} \bar{v}\right\|_{2}^{2}+\left\|\Delta_{H} T_{z}\right\|_{2}^{2}+\left\|\nabla_{H} T_{z z}\right\|_{2}^{2}+\left\|\nabla_{H} \Delta_{H} \eta\right\|_{2}^{2}+\left\|\Delta_{H} \eta_{z}\right\|_{2}^{2}+\left\|\nabla_{H} \Delta_{H} \theta\right\|_{2}^{2}+\left\|\Delta_{H} \theta_{z}\right\|_{2}^{2} .
\end{aligned}
$$

Thus, by (119), we get

$$
\left\|\nabla_{H} v\right\|_{\infty} \leq C\left(\left\|\nabla_{H} \bar{v}\right\|_{H^{1}(M)}+\|T\|_{\infty}+\|\eta\|_{H^{1}(\Omega)}+\|\theta\|_{H^{1}(\Omega)}\right) \log \mathcal{X} .
$$


By virtue of (120), (121), (122), Young's inequality, Cauchy-Schwarz inequality and the above, we obtain

$$
\begin{aligned}
& \frac{d \mathcal{X}}{d t}+C \mathcal{Y} \leq C\|v\|_{2}\left(\left\|\nabla_{H} \bar{v}\right\|_{H^{1}(M)}+\|T\|_{\infty}+\|\eta\|_{H^{1}(\Omega)}+\|\theta\|_{H^{1}(\Omega)}\right) \mathcal{X} \log \mathcal{X} \\
& \quad+\left[1+\|T\|_{\infty}^{4}+\left\|T_{z}\right\|_{2}^{2}+\|\bar{v}\|_{2}^{2}\left(1+\|\bar{v}\|_{H^{1}(M)}^{2}\right)\right. \\
& \left.\quad+\left\|u_{z}\right\|_{H^{1}}^{2}+\left(1+\|\eta\|_{2}^{2}\right)\|\eta\|_{H^{1}}^{2}+\left(1+\|\theta\|_{2}^{2}\right)\|\theta\|_{H^{1}}^{2}\right] \mathcal{X} \\
& \quad+C\left\{\left\|\Delta_{H} Q\right\|_{2}^{2}+\left\|\nabla_{H} Q_{z}\right\|_{2}^{2}+\|\eta\|_{2}^{2}\left\|\nabla_{H} \eta\right\|_{2}^{2}+\|\theta\|_{2}^{2}\left\|\nabla_{H} \theta\right\|_{2}^{2}+\|T\|_{\infty}^{4}+\left\|u_{z}\right\|_{2}^{2}\left\|\nabla_{H} u_{z}\right\|_{2}^{2}\right\}
\end{aligned}
$$

Let $\mathcal{X}=e^{\mathcal{Z}}$. Then, $\frac{d \mathcal{X}}{d t}=e^{\mathcal{Z}} \frac{d \mathcal{Z}}{d t}$. As a result we have

$$
\begin{aligned}
& \frac{d \mathcal{Z}}{d t} \leq C\|v\|_{2}\left(\left\|\nabla_{H} \bar{v}\right\|_{H^{1}(M)}+\|T\|_{\infty}+\|\eta\|_{H^{1}(\Omega)}+\|\theta\|_{H^{1}(\Omega)}\right) \mathcal{Z} \\
& \quad+\left[1+\left\|\Delta_{H} Q\right\|_{2}^{2}+\left\|\nabla_{H} Q_{z}\right\|_{2}^{2}+\|T\|_{\infty}^{4}+\left\|T_{z}\right\|_{2}^{2}+\|\bar{v}\|_{2}^{2}\left(1+\|\bar{v}\|_{H^{1}(M)}^{2}\right)\right. \\
& \left.\quad+\left(1+\left\|u_{z}\right\|_{2}^{2}\right)\left\|u_{z}\right\|_{H^{1}}^{2}+\left(1+\|\eta\|_{2}^{2}\right)\|\eta\|_{H^{1}}^{2}+\left(1+\|\theta\|_{2}^{2}\right)\|\theta\|_{H^{1}}^{2}\right] .
\end{aligned}
$$

Thanks to Gronwall inequality, and the estimates established in the previous subsections, we get

$$
\mathcal{Z} \leq K, \quad \mathcal{X} \leq e^{K}
$$

where

$$
K=e^{C\left(K_{1}+K_{2}+K_{7}\right)}\left[1+\left\|v_{0}\right\|_{H^{4}}^{2}+\left\|T_{0}\right\|_{H^{2}}^{2}+t+\left\|\Delta_{H} Q\right\|_{2}^{2} t+\left\|\nabla_{H} Q_{z}\right\|_{2}^{2} t\right] .
$$

Moreover, we have

$$
\int_{0}^{t} \mathcal{Y} d s \leq K_{8}
$$

where

$$
K_{8}=e^{C\left(K_{1}+K_{2}+K_{7}\right)}\left[1+\left\|v_{0}\right\|_{H^{4}}^{2}+\left\|T_{0}\right\|_{H^{2}}^{2}+t+\left\|\Delta_{H} Q\right\|_{2}^{2} t+\left\|\nabla_{H} Q_{z}\right\|_{2}^{2} t\right] .
$$

Thanks to (103) and the above we conclude that the quantities $\int_{0}^{t}\left\|v_{z z}(s)\right\|_{H^{1}(\Omega)}^{2} d s, \int_{0}^{t}\left\|\Delta_{H} \nabla_{H} v_{z}(s)\right\|_{H^{1}(\Omega)}^{2} d s$, $\left\|\Delta_{H} v_{z}(t)\right\|_{H^{1}(\Omega)},\left\|\nabla_{H} T(t)\right\|_{H^{1}(\Omega)}$, and $\int_{0}^{t}\left\|\nabla_{H} T_{z}(s)\right\|_{H^{1}(\Omega)}^{2} d s$ are all bounded uniformly in time, $t$, over the interval $\left[0, \mathcal{T}_{*}\right)$. Therefore, the strong solution $(v(t), T(t))$ exists globally in time.

\section{Uniqueness of the Solutions}

In this section we state and prove the global existence and uniqueness of the strong solution of system (22)-(28).

Theorem 2. Suppose that $Q \in H^{2}(\Omega)$. Then for every $v_{0} \in H^{4}(\Omega), T_{0} \in H^{2}(\Omega)$ and $\mathcal{T}>0$, there is a unique solution $\left(v, p_{s}, T\right)$ of system $(22)-(28)$ with

$$
\begin{aligned}
& \Delta_{H} v_{z}, \quad \nabla_{H} T \in C\left([0, \mathcal{T}], H^{1}(\Omega)\right), \\
& v_{z z}, \quad \Delta_{H} \nabla_{H} v_{z}, \quad \nabla_{H} T_{z} \in L^{2}\left([0, \mathcal{T}], H^{1}(\Omega)\right),
\end{aligned}
$$

Proof. In Theorem 2 of the previous section we proved that the strong solutions exist globally in time. Therefore, it remains to prove the uniqueness of strong solutions, and their continuous dependence on initial data, in the sense specified by equation (140) below. Let $\left(v_{1},\left(p_{s}\right)_{1}, T_{1}\right)$ and $\left(v_{2},\left(p_{s}\right)_{2}, T_{2}\right)$ be two strong solutions of system $(22)-$ (28) with initial data $\left(\left(v_{0}\right)_{1},\left(T_{0}\right)_{1}\right)$ and $\left(\left(v_{0}\right)_{2},\left(T_{0}\right)_{2}\right)$, respectively. Denote by $\phi=v_{1}-v_{2}, q_{s}=\left(p_{s}\right)_{1}-\left(p_{s}\right)_{2}, \psi=$ 
$T_{1}-T_{2}$. It is clear that

$$
\begin{aligned}
& \frac{\partial \phi}{\partial t}+L_{1} \phi+\left(v_{1} \cdot \nabla_{H}\right) \phi+\left(\phi \cdot \nabla_{H}\right) v_{2}-\left(\int_{-h}^{z} \nabla_{H} \cdot v_{1}(x, y, \xi, t) d \xi\right) \frac{\partial \phi}{\partial z}-\left(\int_{-h}^{z} \nabla_{H} \cdot \phi(x, y, \xi, t) d \xi\right) \frac{\partial v_{2}}{\partial z} \\
& +f_{0} \vec{k} \times \phi+\nabla_{H} q_{s}-\nabla_{H}\left(\int_{-h}^{z} \psi(x, y, \xi, t) d \xi\right)=0, \\
& \frac{\partial \psi}{\partial t}-\frac{1}{R_{3}} \psi_{z z}+v_{1} \cdot \nabla_{H} \psi+\phi \cdot \nabla_{H} T_{2} \\
& -\left(\int_{-h}^{z} \nabla_{H} \cdot v_{1}(x, y, \xi, t) d \xi\right) \frac{\partial \psi}{\partial z}-\left(\int_{-h}^{z} \nabla_{H} \cdot \phi(x, y, \xi, t) d \xi\right)\left(\frac{\partial T_{2}}{\partial z}+\frac{1}{h}\right)=0,
\end{aligned}
$$

with initial data

$$
\begin{aligned}
& \phi(x, y, z, 0)=\left(v_{0}\right)_{1}-\left(v_{0}\right)_{2}, \\
& \psi(x, y, z, 0)=\left(T_{0}\right)_{1}-\left(T_{0}\right)_{2} .
\end{aligned}
$$

Taking the inner product of equation (128) with $\phi$ in $L^{2}(\Omega)$, and equation (129) with $\psi$ in $L^{2}(\Omega)$, we get

$$
\begin{aligned}
& \frac{1}{2} \frac{d\|\phi\|_{2}^{2}}{d t}+\frac{1}{R_{1}}\left\|\nabla_{H} \phi\right\|_{2}^{2}+\frac{1}{R_{2}}\left\|\phi_{z}\right\|_{2}^{2} \\
& =-\int_{\Omega}\left[\left(v_{1} \cdot \nabla_{H}\right) \phi+\left(\phi \cdot \nabla_{H}\right) v_{2}-\left(\int_{-h}^{z} \nabla_{H} \cdot v_{1}(x, y, \xi, t) d \xi\right) \frac{\partial \phi}{\partial z}-\left(\int_{-h}^{z} \nabla_{H} \cdot \phi(x, y, \xi, t) d \xi\right) \frac{\partial v_{2}}{\partial z}\right] \cdot \phi d x d y d z \\
& +\int_{\Omega}\left[f_{0} \vec{k} \times \phi+\nabla_{H} q_{s}-\nabla_{H}\left(\int_{-h}^{z} \psi(x, y, \xi, t) d \xi\right)\right] \cdot \phi d x d y d z,
\end{aligned}
$$

and

$$
\begin{aligned}
& \frac{1}{2} \frac{d\|\psi\|_{2}^{2}}{d t}+\frac{1}{R_{3}}\left\|\psi_{z}\right\|_{2}^{2}=-\int_{\Omega}\left[v_{1} \cdot \nabla_{H} \psi+\phi \cdot \nabla_{H} T_{2}\right. \\
& \left.-\left(\int_{-h}^{z} \nabla_{H} \cdot v_{1}(x, y, \xi, t) d \xi\right) \frac{\partial \psi}{\partial z}-\left(\int_{-h}^{z} \nabla_{H} \cdot \phi(x, y, \xi, t) d \xi\right)\left(\frac{\partial T_{2}}{\partial z}+\frac{1}{h}\right)\right] \psi d x d y d z
\end{aligned}
$$

Notice that

$$
f_{0} \vec{k} \times \phi \cdot \psi=0
$$

Integrating by parts, and using the boundary conditions (25) and (26), we have

$$
\begin{aligned}
& -\int_{\Omega}\left(\left(v_{1} \cdot \nabla_{H}\right) \phi-\left(\int_{-h}^{z} \nabla_{H} \cdot v_{1}(x, y, \xi, t) d \xi\right) \frac{\partial \phi}{\partial z}\right) \cdot \phi d x d y d z=0, \\
& -\int_{\Omega}\left(v_{1} \cdot \nabla_{H} \psi-\left(\int_{-h}^{z} \nabla_{H} \cdot v_{1}(x, y, \xi, t) d \xi\right) \frac{\partial \psi}{\partial z}\right) \cdot \psi d x d y d z=0 .
\end{aligned}
$$

Integrating by parts, and using the boundary conditions (25) and (26), we get

$$
\begin{aligned}
& \int_{\Omega}\left[\nabla_{H} q_{s}-\nabla_{H}\left(\int_{-h}^{z} \psi(x, y, \xi, t) d \xi\right)\right] \cdot \phi d x d y d z \\
& =\int_{\Omega}\left(\int_{-h}^{z} \psi(x, y, \xi, t) d \xi\right)\left(\nabla_{H} \cdot \phi\right) d x d y d z
\end{aligned}
$$


Thus, by (132)-(135) we have

$$
\begin{aligned}
& \frac{1}{2} \frac{d\|\phi\|_{2}^{2}}{d t}+\frac{1}{R_{1}}\left\|\nabla_{H} \phi\right\|_{2}^{2}+\frac{1}{R_{2}}\left\|\phi_{z}\right\|_{2}^{2}=-\int_{\Omega}\left(\phi \cdot \nabla_{H}\right) v_{2} \cdot \phi d x d y d z \\
& +\int_{\Omega} \int_{-h}^{z} \nabla_{H} \cdot \phi(x, y, \xi, t) d \xi \frac{\partial v_{2}}{\partial z} \cdot \phi d x d y d z+\int_{\Omega}\left(\int_{-h}^{z} \psi(x, y, \xi, t) d \xi\right)\left(\nabla_{H} \cdot \phi\right) d x d y d z .
\end{aligned}
$$

and

$$
\begin{aligned}
& \frac{1}{2} \frac{d\|\psi\|_{2}^{2}}{d t}+\frac{1}{R_{3}}\left\|\psi_{z}\right\|_{2}^{2}=-\int_{\Omega}\left(\phi \cdot \nabla_{H} T_{2}\right) \psi d x d y d z \\
& -\int_{\Omega}\left(\nabla_{H} \cdot \phi\right) T_{2} \psi d x d y d z-\int_{\Omega} \int_{-h}^{z} \nabla_{H} \cdot \phi(x, y, \xi, t) d \xi T_{2} \psi_{z} d x d y d z .
\end{aligned}
$$

Notice that by Hölder inequality and (44)

$$
\begin{aligned}
& \left|\int_{\Omega}\left(\phi \cdot \nabla_{H}\right) v_{2} \cdot \phi d x d y d z\right| \leq\left|\int_{\Omega}\right| v_{2}|| \phi|| \nabla_{H} \phi|d x d y d z| \leq\left\|v_{2}\right\|_{6}\|\phi\|_{2}^{\frac{1}{2}}\left\|\nabla_{H} \phi\right\|_{2}^{3 / 2}, \\
& \left|\int_{\Omega} \phi \cdot \nabla_{H} T_{2} \psi d x d y d z\right| \leq\left\|\nabla_{H} T_{2}\right\|_{3}\|\psi\|_{2}\|\phi\|_{6} \leq C\left\|\nabla_{H} T_{2}\right\|_{H^{1}}\|\psi\|_{2}\left\|\nabla_{H} \phi\right\|_{2} ; \\
& \left|\int_{\Omega} \int_{-h}^{z} \nabla_{H} \cdot \phi(x, y, \xi, t) d \xi \frac{\partial v_{2}}{\partial z} \cdot \phi d x d y d z\right| \\
& \leq C\left\|\nabla_{H} \phi\right\|_{2}|| \frac{\partial v_{2}}{\partial z}\left\|_{6}\right\| \phi\left\|_{3} \leq C|| \frac{\partial v_{2}}{\partial z}\right\|\left\|_{6}\right\| \phi\left\|_{2}^{\frac{1}{2}}\right\| \nabla_{H} \phi \|_{2}^{3 / 2} ; \\
& \left|\int_{\Omega} \int_{-h}^{z} \nabla_{H} \cdot \phi(x, y, \xi, t) d \xi \frac{\partial T_{2}}{\partial z} \psi d x d y d z\right| \leq\left|\int_{\Omega} \int_{-h}^{0}\right| \nabla_{H} \phi\left|d z \int_{-h}^{0}\right| \frac{\partial T_{2}}{\partial z} \psi|d z d x d y| \\
& \leq C\left|\int_{\Omega} \int_{-h}^{0}\right| \nabla_{H} \phi\left|d z\left(\int_{-h}^{0}\left|\frac{\partial T_{2}}{\partial z}\right|^{2} d z\right)^{1 / 2}\left(\int_{-h}^{0}|\psi|^{2} d z\right)^{1 / 2} d x d y\right| \\
& \leq C\left\|\int_{-h}^{0}\left|\frac{\partial T_{2}}{\partial z}\right|^{2} d z\left|\|\|_{\infty}^{1 / 2}\left\|\nabla_{H} \phi\right\|_{2}\|\psi\|_{2} \leq C\right| \frac{\partial \Delta_{H} T_{2}}{\partial z}\right\|\left\|_{2}\right\| \nabla_{H} \phi\left\|_{2}\right\| \psi \|_{2} .
\end{aligned}
$$

Therefore, by estimates (136)-(139), we reach

$$
\begin{aligned}
& \frac{1}{2} \frac{d\left(\|\phi\|_{2}^{2}+\|\psi\|_{2}^{2}\right)}{d t}+\frac{1}{R_{1}}\left\|\nabla_{H} \phi\right\|_{2}^{2}+\frac{1}{R_{2}}\left\|\phi_{z}\right\|_{2}^{2}+\frac{1}{R_{3}}\left\|\psi_{z}\right\|_{2}^{2} \\
& \leq C\left(\left\|v_{2}\right\|_{6}\|\phi\|_{2}^{\frac{1}{2}}\left\|\nabla_{H} \phi\right\|_{2}^{3 / 2}+\left\|\nabla_{H} T_{2}\right\|_{H^{1}}\|\psi\|_{2}\left\|\nabla_{H} \phi\right\|_{2}+\left\|\partial_{z} v_{2}\right\|_{6}\|\phi\|_{2}^{\frac{1}{2}}\left\|\nabla_{H} \phi\right\|_{2}^{3 / 2}\right)+C\left\|\frac{\partial \Delta_{H} T_{2}}{\partial z}\right\|_{2}\left\|\nabla_{H} \phi\right\|_{2}\|\psi\|_{2} .
\end{aligned}
$$

By Young's inequality and the Cauchy-Schwarz inequality, we get

$$
\frac{d\|\phi\|_{2}^{2}+\|\psi(t)\|_{2}^{2}}{d t} \leq C\left(\left\|v_{2}\right\|_{6}^{4}+\left\|\nabla_{H} T_{2}\right\|_{H^{1}}^{2}+\left\|\partial_{z} v_{2}\right\|_{6}^{4}+\left\|\frac{\partial \Delta_{H} T_{2}}{\partial z}\right\|_{2}^{2}\right)\left(\|\phi\|_{2}^{2}+\|\psi\|_{2}^{2}\right) .
$$

Thanks to Gronwall inequality, we obtain

$$
\begin{aligned}
& \|\phi(t)\|_{2}^{2}+\|\psi(t)\|_{2}^{2} \leq\left(\|\phi(t=0)\|_{2}^{2}+\|\psi(t=0)\|_{2}^{2}\right) \times \\
& \quad \exp \left\{C \int_{0}^{t}\left(\left\|v_{2}(s)\right\|_{6}^{4}+\left\|\nabla_{H} T_{2}(s)\right\|_{H^{1}}^{2}+\left\|\partial_{z} v_{2}(s)\right\|_{6}^{4}+\left\|\frac{\partial \Delta_{H} T_{2}}{\partial z}\right\|_{2}^{2}\right) d s\right\} .
\end{aligned}
$$


As a result of (124), we have

$$
\|\phi(t)\|_{2}^{2}+\|\psi(t)\|_{2}^{2} \leq\left(\|\phi(t=0)\|_{2}^{2}+\|\psi(t=0)\|_{2}^{2}\right) \exp \left\{C\left(\left(K_{3}^{2 / 3}+K+K_{4}^{2 / 3}\right) t+K_{8}\right)\right\} .
$$

The above inequality proves the continuous dependence of the solutions on the initial data, and in particular, when $\phi(t=0)=0$ and $\psi(t=0)=0$, we have $\phi(t)=0$ and $\psi(t)=0$, for all $t \geq 0$. Therefore, the strong solution is unique.

\section{ACKNOWLEDGMENTS}

The authors are thankful to the kind hospitality of the Institute for Mathematics and its Applications (IMA), University of Minnesota, where part of this work was completed. This work was supported in part by the NSF grants no. DMS-0709228, DMS-0708832 and DMS-1009950. The work of C.C. was also supported in part by the Florida International University Foundation. E.S.T. also acknowledges the warm hospitality of the Freie Universität - Berlin and the partial support of the Alexander von Humboldt Stiftung/Foundation and the Minerva Stiftung/Foundation.

\section{REFERENCES}

[1] R.A. Adams, Sobolev Spaces, Academic Press, New York, 1975.

[2] J. T. Beale, T. Kato and A. Majda, Remarks on the breakdown of smooth solutions for the 3-D Euler equations, Comm. Math. Phys. 94 (1984), 61-66.

[3] H. Bénard, Les tourbillons cellulaires dans une nappe liquide transportant de la chaleur par convection en régime permanent, Ann.d. Chimie et de Physique, 23 (1901), 62-144.

[4] H. Brézis and T. Gallouet, Nonlinear Schrödinger evolution equations, Nonlinear Anal. 4 (1980), 677-681.

[5] H. Brézis and S. Wainger, Note on limiting cases of Sobolev embeddings and convolution inequalities, Comm. Partial Differential Equations 5 (1980), 773-789.

[6] C. Cao and E.S. Titi, Global well-posedness and finite dimensional global attractor for a 3-D planetary geostrophic viscous model, Comm. Pure Appl. Math. 56, 198-233, 2003

[7] C. Cao and E.S. Titi, Global well-posedness at large for the 3D viscous primitive equations of ocean dynamics, Ann. of Math., 166 (2007), 245-267.

[8] B. Castaing, G. Gunaratne, F. Heslot, L. Kadanoff, A. Libchaber, S. Thomae, X. Z. Wu, S. Zaleski and G. Zanetti, Scaling of hard thermal turbulence in Rayleigh-Benard convection, J. Fluid Mech. 204(1989), 1-29.

[9] D. Chae, Global regularity for the 2D Boussinesq equations with partial viscosity terms, Adv. Math. 203 (2006), $497-513$.

[10] P. Constantin and C. Foias, Navier-Stokes Equations, The University of Chicago Press, 1988.

[11] R. Danchin and M. Paicu, Global existence results for the anisotropic Boussinesq system in dimension two, arXiv: 0809.4984v1 [math.AP] 19 Sep 2008.

[12] W. E and C. Shu, Small-scale structures in Boussinesq convection, Phys. Fluids 6 (1994), 49-58.

[13] H. Engler, An alternative proof of the Brezis-Wainger inequality, Comm. Partial Differential Equations 14 (1989), 541-544.

[14] C. Foias, O.P. Manley and R. Temam, Attractors for the Bénard problem: existence and physical bounds on their fractal dimension, Nonlinear Anal. 11 (1987), 939-967.

[15] G.P. Galdi, An Introduction to the Mathematical Theory of the Navier-Stokes Equations, Vol. I \& II, Springer-Verlag, 1994.

[16] A.E. Gargett, Vertical eddy diffusivity in the ocean interior, J. Mar. Res., 42 (1984), 359-393.

[17] C.J.R. Garrett, Oceanography: A stirring tale of mixing, Nature, 364 (1993), 670-671.

[18] A.V. Getling, Rayleigh Benard Convection: Structures and Dynamics, World Scientific, 1998.

[19] F. Guillén-Gonzáez, N. Masmoudi and M.A. Rodríguez-Bellido, Anisotropic estimates and strong solutions of the primitive equations, Differential and Intergral Equations 14 no. 1 (2001), 1381-1408.

[20] T. Hou and C. Li, Global well-posedness of the viscous Boussinesq equations, Discrete Contin. Dyn. Syst. 12 (2005), 1-12.

[21] T. Kato, Remarks on the Euler and Navier-Stokes equations in $\mathbb{R}^{2}$, Nonlinear functional analysis and its applications, Proc. Summer Res. Inst., Berkeley/Calif. 1983, Proc. Symp. Pure Math. 45 (1986), 1-7.

[22] I. Kukavica and M. Ziane, On the regularity of the primitive equations of the ocean, Nonlinearity 20 (2007), $2739-2753$.

[23] O.A. Ladyzhenskaya, The Boundary Value Problems of Mathematical Physics, Springer-Verlag, 1985.

[24] J.L. Lions, R. Temam and S. Wang, New formulations of the primitive equations of atmosphere and applications, Nonlinearity 5 (1992), 237-288. 
[25] J.L. Lions, R. Temam and S. Wang, On the equations of the large scale Ocean, Nonlinearity 5 (1992), 1007-1053.

[26] A. Majda and A.L. Bertozzi, Vorticity and Incompressible Flow, Cambridge University Press, 2002.

[27] C. Marchioro and M. Pulvirenti, Mathematical Theory of Incompressible Nonviscous Fluids, Applied Mathematical Sciences 96, Springer, New York, 1994.

[28] J.J. Niemela, L. Skrbek, K.R. Sreenivasan and R.J. Donnelly, Turbulent convection at very high Rayleigh numbers, Nature 404(2000), 837-840.

[29] J. Pedlosky, Geophysical Fluid Dynamics, Springer-Verlag, New York, 1987.

[30] L. Rayleigh, On convection currents in a horizontal layer of fluid, when the higher temperature is on th under side, Phil. Mag. 6 (1916), 529-546.

[31] L.F. Richardson, Weather Prediction by Numerical Process, Cambridge University Press, Cambridge 1922 (reprint, Dover, New York, 1988).

[32] R. Salmon, Lectures on Geophysical Fluid Dynamics, Oxford University Press, New York, Oxford, 1998.

[33] R. Samelson and G. Vallis, A simple friction and diffusion scheme for planetary geostrophic basin models, J. Phys. Oceanogr., 27 (1997), 186-194.

[34] H. Sohr, The Navier-Stokes Equations, An Elementary Functional Analytic Approach, Birkhäuser Verlag, Basel, 2001.

[35] R. Temam, Navier-Stokes Equations, Theory and Numerical Analysis, North-Holland, 1984.

[36] R. Temam, Infinite-Dimensional Dynamical Systems in Mechanics and Physics, Applied Mathematical Sciences, Vol. 68, Springer-Verlag, New York, 1988.

[37] R. Temam and M. Ziane, Some mathematical problems in geophysical fluid dynamics, Handbook of Mathematical Fluid Dynamics. Vol. III, 535-657, North-Holland, Amsterdam, 2004.

[38] G.K. Vallis, Atmospheric and Oceanic Fluid Dynamics, Cambridge University Press, 2006

[39] V.I. Yudovitch, Non-stationary flow of an ideal incompressible liquid, Zh. Vychisl. Mat. i Mat. Fiz. 6 (1963), $1032-1066$.

(C. Cao) Department of Mathematics, Florida International University, University Park, Miami, FL 33199, USA.

E-mail address: caoc@fiu.edu

(E.S. Titi) Department of Mathematics, and Department of Mechanical and Aerospace Engineering, University of California, Irvine, CA 92697-3875, USA. Also: Department of Computer Science and Applied Mathematics, Weizmann Institute of Science, Rehovot 76100, IsRael.

E-mail address: etiti@math.uci.edu

E-mail address: edriss.titi@weizmann.ac.il 